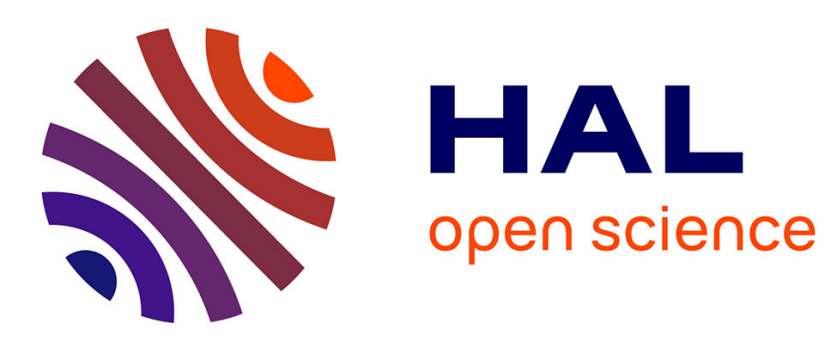

\title{
Modeling and Control of Legged Robots
}

\author{
Pierre-Brice Wieber, Russ Tedrake, Scott Kuindersma
}

\section{To cite this version:}

Pierre-Brice Wieber, Russ Tedrake, Scott Kuindersma. Modeling and Control of Legged Robots. Springer Handbook of Robotics, Springer International Publishing, pp.1203-1234, 2016, 10.1007/9783-319-32552-1_48. hal-02487855

\section{HAL Id: hal-02487855 \\ https://hal.inria.fr/hal-02487855}

Submitted on 21 Feb 2020

HAL is a multi-disciplinary open access archive for the deposit and dissemination of scientific research documents, whether they are published or not. The documents may come from teaching and research institutions in France or abroad, or from public or private research centers.
L'archive ouverte pluridisciplinaire HAL, est destinée au dépôt et à la diffusion de documents scientifiques de niveau recherche, publiés ou non, émanant des établissements d'enseignement et de recherche français ou étrangers, des laboratoires publics ou privés. 


\section{Chapter 48}

\section{Modeling and Control of Legged Robots}

\section{Summary}

\section{Introduction}

The promise of legged robots over standard wheeled robots is to provide improved mobility over rough terrain. This promise builds on the decoupling between the environment and the main body of the robot that the presence of articulated legs allows, with two consequences. First, the motion of the main body of the robot can be made largely independent from the roughness of the terrain, within the kinematic limits of the legs: legs provide an active suspension system. Indeed, one of the most advanced hexapod robots of the 1980s was aptly called the Adaptive Suspension Vehicle [1]. Second, this decoupling allows legs to temporarily leave their contact with the ground: isolated footholds on a discontinuous terrain can be overcome, allowing to visit places absolutely out of reach otherwise. Note that having feet firmly planted on the ground is not mandatory here: skating is an equally interesting option, although rarely approached so far in robotics.

Unfortunately, this promise comes at the cost of a hindering increase in complexity. It is only with the unveiling of the Honda P2 humanoid robot in 1996 [2], and later of the Boston Dynamics BigDog quadruped robot in 2005 that legged robots finally began to deliver real-life capabilities that are just beginning to match the long sought animal-like mobility over rough terrain. Not matching yet the capabilities of humans and animals, legged robots do contribute however already to understanding their locomotion, as evidenced by the many fruitful collaborations between robotics and biomechanics researchers. 


\subsection{A Brief History of Legged Robots}

Before the advent of digital computers, legged machines could be approached only by electro-mechanical means, lacking in advanced feedback control. This "pre-robotics" period culminated with the General Electric Walking Truck developed by Ralph Mosher, which inspired awe in the mid 1960s. The limb motions of this elephant-size quadruped machine were directly reflecting the limb motions of the onboard operator, who was responsible for all motion control and synchronization. Unfortunately, the strenuous concentration that this required limited operation to less than 15 minutes.

Digitally controlled legged robots started to appear in the late 1960s. Among early pioneers, Robert McGhee initiated a series of quadruped and hexapod robots first at University of South California, then at Ohio State University, culminating in the mid 1980s with the Adaptive Suspension Vehicle, a human carrying hexapod vehicle walking on natural and irregular outdoor terrain [1], while Ichiro Kato initiated a long series of biped and humanoid robots in the Waseda University, a series still continuing nearly half a century later [3]. But by the end of the $1970 \mathrm{~s}$, all legged robots were still limited to quasi-static gaits, i.e. slow walking motions with the Center of Mass of the robot always kept above its feet.

The transition to dynamic legged locomotion occurred in the beginning of the 1980s, with the first dynamically walking bipedal robot demonstrated at the Tokyo University [4], and the famous series of hopping and running monopedal, bipedal and quadrupedal robots developed at the MIT LegLab under the direction of Marc Raibert [5]. Key theoretical breakthroughs came in the end of the 1980s, when Tad McGeer demonstrated that stable dynamic walking motions could be obtained by pure mechanical means, giving rise to a whole new field of research, passive dynamic walking, introducing new, key concepts such as orbital stability using Poincaré maps [6], with one simple conclusion: you need not have complete (or any) control to be able to walk dynamically and efficiently.

Legged robots were still mostly research laboratory curiosities working in limited situations when Honda unveiled the P2 humanoid robot in 1996 [2], a decade long secret project demonstrating unprecedented versatility and robustness, followed in 2000 by the Asimo humanoid robot. The world of humanoid and legged robots was ripe for companies to begin investing. A handful of other Japanese companies such as Toyota or Kawada were quick to follow with their own humanoid robots, while Sony began selling more than 150,000 of its Aibo home companion robot dogs. Boston Dynamics, a company Marc Raibert founded after leaving the MIT LegLab, finally unveiled its BigDog quadruped robot in 2005 [7], which was the first to demonstrate true animal-like locomotion capabilities on rough terrain.

The progress over the last decades has been remarkable. Profound questions have finally been answered: we now understand how to make legged robots walk and run dynamically. But other profound questions still have to be answered, such as how best to make them walk and run efficiently. The performance of 
legged robots needs to be improved in many ways: energy, speed, reactivity, versatility, robustness, etc. We will therefore discuss in this Chapter how legged robots are usually modeled in Section 48.2 and how dynamic motions are currently generated and controlled in Sections 48.4 and 48.5, before discussing in Section 48.6 the current trends in improving their efficiency.

\subsection{The dynamics of legged locomotion}

One of the major difficulties in making a legged robot walk or run is keeping its balance: where should the robot place its feet, how should it move its body in order to move safely in a given direction, even in case of strong perturbations? This difficulty comes from the fact that contact forces with the environment are an absolutely necessity to generate and control locomotion, but they are limited by the mechanical laws of unilateral contact.

This essential role of the contact forces is particularly clear in the derivatives of the total linear and angular momenta of the robot, the former involving the motion of its Center of Mass. Because of the importance of contact forces for legged locomotion, we briefly discuss here their different models.

\subsubsection{Lagrangian dynamics}

Structure of the configuration space. As for every robot moving in their $3 \mathrm{D}$ environment (in space or underwater for example), the configuration space of legged robots combines the configuration $\hat{\boldsymbol{q}} \in \mathbb{R}^{N}$ of their $N$ joints with a global position $\boldsymbol{x}_{0} \in \mathbb{R}^{3}$ and orientation $\boldsymbol{\theta}_{0} \in \mathbb{R}^{3}$ (representing an element of $S O(3))$ :

$$
\boldsymbol{q}=\left[\begin{array}{c}
\hat{\boldsymbol{q}} \\
\boldsymbol{x}_{0} \\
\boldsymbol{\theta}_{0}
\end{array}\right]
$$

The position $\boldsymbol{x}_{0}$ and orientation $\boldsymbol{\theta}_{0}$ are typically those of a central body (pelvis or trunk) or of an extremity (foot or hand).

Structure of the Lagrangian dynamics. The specific structure of the configuration space outlined above is naturally reflected in the Lagrangian dynamics

$$
\boldsymbol{M}(\boldsymbol{q})\left(\left[\begin{array}{c}
\ddot{\hat{\boldsymbol{q}}} \\
\ddot{\boldsymbol{x}}_{0} \\
\ddot{\boldsymbol{\theta}}_{0}
\end{array}\right]+\left[\begin{array}{l}
\mathbf{0} \\
\boldsymbol{g} \\
\mathbf{0}
\end{array}\right]\right)+\boldsymbol{n}(\boldsymbol{q}, \dot{\boldsymbol{q}})=\left[\begin{array}{l}
\boldsymbol{u} \\
\mathbf{0} \\
\mathbf{0}
\end{array}\right]+\sum_{i} \boldsymbol{C}_{i}(\boldsymbol{q})^{\top} \boldsymbol{f}_{i}
$$

of the system, where $\boldsymbol{M}(\boldsymbol{q}) \in \mathbb{R}^{(N+6) \times(N+6)}$ is the generalized inertia matrix of the robot, $-\boldsymbol{g} \in \mathbb{R}^{3}$ is the constant gravity acceleration vector, $\boldsymbol{n}(\boldsymbol{q}, \dot{\boldsymbol{q}}) \in \mathbb{R}^{N+6}$ is the vector of Coriolis and centrifugal effects, $\boldsymbol{u} \in \mathbb{R}^{N}$ is the vector of joint torques, and for all $i, \boldsymbol{f}_{i} \in \mathbb{R}^{3}$ is a force exerted by the environment on the robot and $\boldsymbol{C}_{i}(\boldsymbol{q}) \in \mathbb{R}^{(N+6) \times 3}$ is the associated Jacobian matrix [8]. 
Since the vector $\boldsymbol{u}$ of joint torques has the same size as the vector $\hat{\boldsymbol{q}}$ of joint positions, the whole dynamics including the global position $\boldsymbol{x}_{0}$ and orientation $\boldsymbol{\theta}_{0}$ appears to be underactuated if no external forces $\boldsymbol{f}_{i}$ are exerted.

\subsubsection{Newton and Euler equations of motion}

Center of Mass and Angular Momentum. A consequence of the structure (48.2) of the Lagrangian dynamics is that the part of this dynamics which is not directly actuated involves the Newton and Euler equations of motion of the robot taken as a whole (see [8] for detailed derivations). The Newton equation can be written in the following way:

$$
m(\ddot{\boldsymbol{c}}+\boldsymbol{g})=\sum_{i} \boldsymbol{f}_{i}
$$

with $m$ the total mass of the robot and $\boldsymbol{c}$ the position of its Center of Mass $(\mathrm{CoM})$. The Euler equation can be expressed with respect to the CoM in the following way:

$$
\dot{\boldsymbol{L}}=\sum_{i}\left(\boldsymbol{p}_{i}-\boldsymbol{c}\right) \times \boldsymbol{f}_{i}
$$

with $\boldsymbol{p}_{i}$ the points of applications of the forces $\boldsymbol{f}_{i}$ and

$$
\boldsymbol{L}=\sum_{k}\left(\boldsymbol{x}_{k}-\boldsymbol{c}\right) \times m_{k} \dot{\boldsymbol{x}}_{k}+\boldsymbol{I}_{k} \boldsymbol{\omega}_{k}
$$

the angular momentum of the whole robot with respect to its CoM, with $\dot{\boldsymbol{x}}_{k}$ and $\boldsymbol{\omega}_{k}$ the translation and rotation velocities of the different parts $k$ of the robot, $m_{k}$ and $\boldsymbol{I}_{k}$ their masses and inertia tensor matrices (expressed in global coordinates).

The Newton equation makes it obvious that the robot needs external forces, $\boldsymbol{f}_{i}$, in order to move its CoM in a direction other than that of gravity. The Euler equation is more subtle, as we will see during flight phases.

Flight phases. During flight phases, when a legged robot is not in contact with its environment, not experiencing any contact forces, $\boldsymbol{f}_{i}$, the Newton equation (48.3) simplifies to

$$
\ddot{\boldsymbol{c}}=-\boldsymbol{g} \text {. }
$$

In this case, the CoM invariably accelerates along the gravity vector $-\boldsymbol{g}$ with constant horizontal speed, following a standard falling motion: there is absolutely no possibility to control the CoM to move in any different way. The Euler equation (48.4) simplifies in the same way to

$$
\dot{\boldsymbol{L}}=0
$$

imposing a conservation of the angular momentum, $\boldsymbol{L}$. In this case, however, the robot is still able to generate and control both joint motions and global 


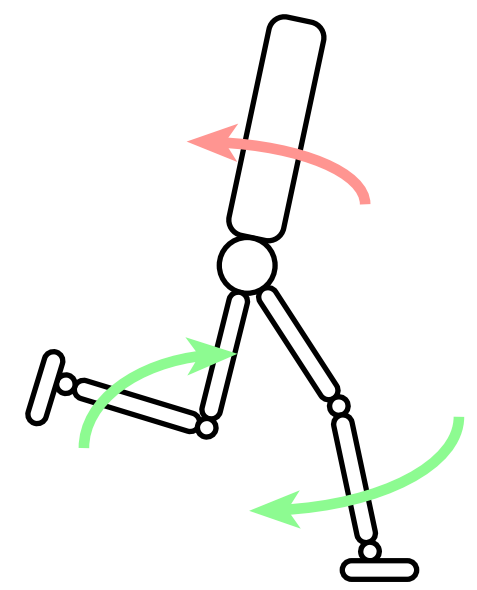

Figure 48.1: Even though the angular momentum is constant during flight phases, the robot is still able to generate and control rotations of the whole body (in red) with the help of leg or arm motions (in green), as a result of the non-holonomy of the angular momentum. This is how cats fall back on their feet when dropped from any initial orientation.

rotations, this is how cats are able to fall back on their feet when dropped from any initial orientation (Figure 48.1). This is a result of the non-holonomy of the angular momentum (48.5) which is not the derivative of any function of the configuration of the robot [9]. As a result, even though the angular momentum, $\boldsymbol{L}$, is kept constant during the whole flight phase, the joint configuration, $\hat{\boldsymbol{q}}$, and the global orientation, $\boldsymbol{\theta}_{0}$, of the robot can be driven to any desired value at the end of the flight phase. We will see in Section 48.5 how this impacts the control of legged robots. Note that the dynamics of legged robots during flight phases is similar to the dynamics of free-floating space robots discussed in Chapter 55. Further discussion and developments can be found there.

In contact with a flat ground: the Center of Pressure. In case the forces applied by the environment on the robot are due to contacts with a flat ground (while standing still, walking or running), let us consider a reference frame oriented along the ground, with the $\boldsymbol{z}$ axis orthogonal to it (therefore tilted if the ground is tilted, see Figure 48.5). Without loss of generality, let us suppose that the points of contact, $\boldsymbol{p}_{i}$, with the ground are all such that $p_{i}^{z}=0$.

Let us consider then the sum of the Euler equation (48.4) and the cross product of the CoM, $\boldsymbol{c}$, with the Newton equation (48.3):

$$
m \boldsymbol{c} \times(\ddot{\boldsymbol{c}}+\boldsymbol{g})+\dot{\boldsymbol{L}}=\sum_{i} \boldsymbol{p}_{i} \times \boldsymbol{f}_{i}
$$


and let us divide the result by the $z$ coordinate of the Newton equation to obtain

$$
\frac{m \boldsymbol{c} \times(\ddot{\boldsymbol{c}}+\boldsymbol{g})+\dot{\boldsymbol{L}}}{m\left(\ddot{c}^{z}+g^{z}\right)}=\frac{\sum_{i} \boldsymbol{p}_{i} \times \boldsymbol{f}_{i}}{\sum_{i} f_{i}^{z}} .
$$

Since $p_{i}^{z}=0$, the $x$ and $y$ coordinates of this equation can be simplified in the following way:

$$
\boldsymbol{c}^{x, y}-\frac{c^{z}}{\ddot{c}^{z}+g^{z}}\left(\ddot{\boldsymbol{c}}^{x, y}+\boldsymbol{g}^{x, y}\right)+\frac{1}{m\left(\ddot{c}^{z}+g^{z}\right)} \boldsymbol{S} \dot{\boldsymbol{L}}^{x, y}=\frac{\sum_{i} f_{i}^{z} \boldsymbol{p}_{i}^{x, y}}{\sum_{i} f_{i}^{z}}
$$

with a simple rotation matrix $\boldsymbol{S}=\left[\begin{array}{cc}0 & -1 \\ 1 & 0\end{array}\right]$.

On the right hand side appears the definition of the Center of Pressure $(\mathrm{CoP}), \boldsymbol{z}$, of the contact forces, $\boldsymbol{f}_{i}$. These contact forces are usually unilateral (the robot can push on the ground, not pull):

$$
f_{i}^{z} \geq 0
$$

which implies that the $\mathrm{CoP}$ is bound to lie in the convex hull of the contact points (Figure 48.2):

$$
\boldsymbol{z}^{x, y}=\frac{\sum_{i} f_{i}^{z} \boldsymbol{p}_{i}^{x, y}}{\sum_{i} f_{i}^{z}} \in \operatorname{conv}\left\{\boldsymbol{p}_{i}^{x, y}\right\}
$$

Combining this inclusion with the dynamic equation (48.10) reveals an Ordinary Differential Inclusion (ODI)

$$
\boldsymbol{c}^{x, y}-\frac{c^{z}}{\ddot{c}^{z}+g^{z}}\left(\ddot{\boldsymbol{c}}^{x, y}+\boldsymbol{g}^{x, y}\right)+\frac{1}{m\left(\ddot{c}^{z}+g^{z}\right)} \boldsymbol{S} \dot{\boldsymbol{L}}^{x, y}=\boldsymbol{z}^{x, y} \in \operatorname{conv}\left\{\boldsymbol{p}_{i}^{x, y}\right\}
$$

which bounds the motion of the CoM, $\boldsymbol{c}$, of the robot and the variations of its angular momentum, $\boldsymbol{L}$, with respect to the position, $\boldsymbol{p}_{i}^{x, y}$, of the contact points.

This ODI can be reorganized in the following way:

$$
\frac{c^{z}}{\ddot{c}^{z}+g^{z}}\left(\ddot{\boldsymbol{c}}^{x, y}+\boldsymbol{g}^{x, y}\right)=\left(\boldsymbol{c}^{x, y}-\boldsymbol{z}^{x, y}\right)+\frac{1}{m\left(\ddot{c}^{z}+g^{z}\right)} \boldsymbol{S} \dot{\boldsymbol{L}}^{x, y}
$$

in order to expose its simple geometric meaning in Figure 48.3. We can see especially that aside from the effects of gravity, $\boldsymbol{g}^{x, y}$, and variations, $\dot{\boldsymbol{L}}^{x, y}$, of the angular momentum, the horizontal acceleration, $\ddot{\boldsymbol{c}}^{x, y}$, of the CoM is the result of a force pushing the CoM, $\boldsymbol{c}^{x, y}$, away from the $\mathrm{CoP}, \boldsymbol{z}^{x, y}$, which is bound to lie in the convex hull of the contact points. We have here an intrinsically unstable dynamics.

Note finally that the definition (48.12) of the CoP can be reorganized to show that the horizontal momenta of the contact forces, $\boldsymbol{f}_{i}$, with respect to the $\mathrm{CoP} \boldsymbol{z}$ are equal to zero:

$$
\left[\sum_{i}\left(\boldsymbol{p}_{i}-\boldsymbol{z}\right) \times \boldsymbol{f}_{i}\right]^{x, y}=\sum_{i}\left(\boldsymbol{p}_{i}^{x, y}-\boldsymbol{z}^{x, y}\right) f_{i}^{z}=0 .
$$

Hence the CoP is also referred to as the Zero Moment Point (ZMP) [10, 11]. 


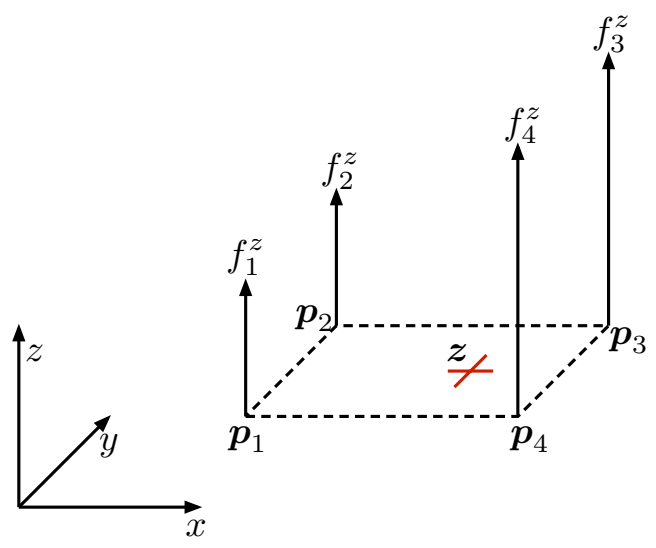

Figure 48.2: The CoP, $\boldsymbol{z}$, is bound to lie in the convex hull of contact points, $p_{i}$.

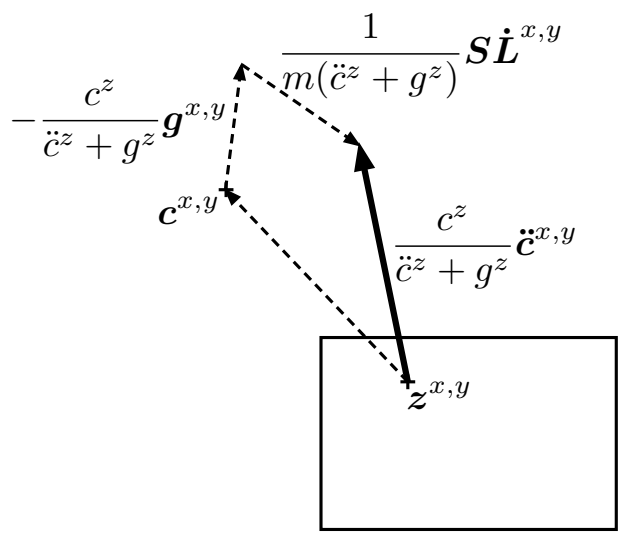

Figure 48.3: The horizontal acceleration, $\ddot{\boldsymbol{c}}^{x, y}$, of the CoM of the robot is the sum of a force pushing the CoM, $\boldsymbol{c}^{x, y}$, away from the CoP, $\boldsymbol{z}^{x, y}$, the effect of gravity, $-\boldsymbol{g}^{x, y}$, and variations, $\dot{\boldsymbol{L}}^{x, y}$, of the angular momentum. 
In contact with multiple surfaces. If the contact points, $\boldsymbol{p}_{i}$, are not all on the same plane, we can introduce a $\mathrm{CoP}$ for each contact surface, but we cannot introduce a unique $\mathrm{CoP}$ for all contact forces as we did previously. Approximations, and generalizations to limited multiple contact situations have been proposed but haven't been widely adopted $[11,12,13,14]$. In the general case, the Newton and Euler equations (48.3) and (48.4) have to be considered explicitly together with the unilaterality condition (48.11) in order to check which motion is feasible or not $[15,16,17]$. The problem we have to solve then relates very closely to the force closure problem discussed in Chapter 38 on Grasping. Different ways to solve it as quickly as possible have been proposed [15, 18], and used mostly so far to measure offline the stability robustness of a given motion [19, 20], and only recently for motion planning, once again offline [21]. Refinements to curved contact surfaces have also been proposed [22].

\subsubsection{Contact models}

The structure of the Lagrangian dynamics makes it clear that contact forces are central to the modeling and control of legged robots. But note that the only characteristics of these forces that we have introduced so far is their unilaterality (48.11). As a result, the previous analysis applies to various contact situations: walking and running motions, but also sliding situations such as when skiing or skating, or even rolling situations [23, 24].

Let us briefly discuss now standard contact models (more details can be found in Chapter 37 on Contact Modeling and Manipulation). Concerning motion and forces tangential to the contact surfaces, a simple Coulomb friction model is usually considered. Concerning motion and forces orthogonal to the contact surfaces, two options are generally considered: a compliant or a rigid model, introducing impacts and other nonsmooth behaviors.

Coulomb friction. When a contact point, $\boldsymbol{p}_{i}$, is sliding on its contact surface, the corresponding tangential contact force, $\boldsymbol{f}_{i}^{x, y}$, is proportional to the normal force, $f_{i}^{z}$, in a direction opposite to the sliding motion:

$$
\boldsymbol{f}_{i}^{x, y}=-\mu_{0} f_{i}^{z} \frac{\dot{\boldsymbol{p}}_{i}^{x, y}}{\left\|\dot{\boldsymbol{p}}_{i}^{x, y}\right\|} \text { if } \dot{\boldsymbol{p}}_{i}^{x, y} \neq 0,
$$

with $\mu_{0}>0$ the friction coefficient. When the contact point is sticking and not sliding, the norm of the tangential force is simply bounded, with the same friction coefficient:

$$
\left\|\boldsymbol{f}_{i}^{x, y}\right\| \leq \mu_{0} f_{i}^{z} \text { if } \dot{\boldsymbol{p}}_{i}^{x, y}=0 .
$$

This is typically referred to as the friction cone. Note that this friction model directly implies the unilaterality condition (48.11).

Compliant contact models. Compliant contact models take into account the visco-elastic properties of the materials in contact in the direction orthogonal 
to the contact surfaces:

$$
f_{i}^{z}=-K_{i} p_{i}^{z}-\Lambda_{i} \dot{p}_{i}^{z} \text { if } p_{i}^{z} \leq 0,
$$

where $K_{i}$ and $\Lambda_{i}$ are stiffness and damping coefficients, respectively. In this case, the normal force, $f_{i}^{z}$, appears to be the result of a penetration of the contact point below the contact surface, i.e. when $p_{i}^{z}<0$. Note that, as it is here, this model doesn't satisfy the unilaterality condition (48.11) in all situations and needs therefore to be saturated to enforce this property. Of course, when there is no contact, there is no contact force:

$$
f_{i}^{z}=0 \text { if } p_{i}^{z}>0 .
$$

A similar spring-damper model can also be used to determine the frictional contact force, $f_{i}^{x, y}$, subject to the bounds of the friction cone (48.17).

Rigid contact models. Rigid contact models are somewhat simpler to introduce: either there is contact and the normal force can take any non-negative value,

$$
f_{i}^{z} \geq 0 \text { if } p_{i}^{z}=0
$$

or there is no contact and no contact force:

$$
f_{i}^{z}=0 \text { if } p_{i}^{z}>0 .
$$

In this case, no penetration of the contact points below the contact surfaces are considered. Note also that the unilaterality condition (48.11) is satisfied here by definition. As a matter of fact, this rigid model can be summarized in the following way:

$$
f_{i}^{z} \geq 0, p_{i}^{z} \geq 0, f_{i}^{z} p_{i}^{z}=0,
$$

where it appears that both the normal force, $f_{i}^{z}$, and the position of the contact point with respect to the contact surface, $p_{i}^{z}$, must be non-negative, but at least one of them must be equal to 0 . This is called a complementarity condition. Analogous to equations (48.18)-(48.19), it defines the normal force to be either zero, or the force necessary to maintain $p_{i}^{z}=0$. Note that the sticking-or-sliding behavior of Coulomb friction can also be represented with such a complementarity condition. Note also that this set of implicit equations may not have a solution or may have multiple ones in certain pathologic situations [25].

Rigid and compliant contact models are compared in Figure 48.4 in a static situation (when $\dot{p}_{i}^{z}=0$ ), showing clearly how the rigid model corresponds to an infinitely stiff compliant model. Compliant contact models can model more accurately the deformation of the contacting bodies, but accurate models often require very stiff springs, causing the resulting differential equations to be numerically stiff, what can slow down or complicate numerical analysis. Rigid models are comparatively more straightforward to integrate and analyze, with purely rigid bodies and contact surfaces. They are therefore preferred when theoretically or numerically studying the stability of legged robots, when optimizing walking or running trajectories, etc. 


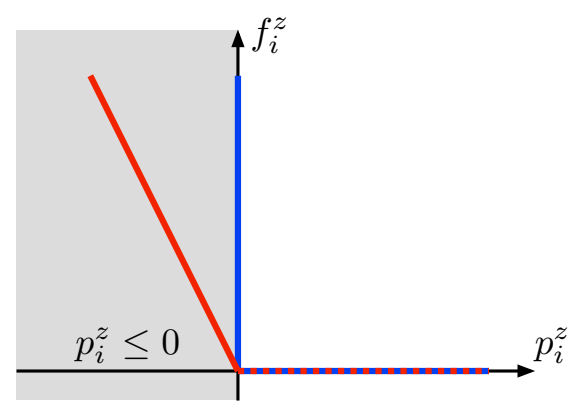

Figure 48.4: Comparison of a rigid (blue) and compliant (red) contact model in a static situation. Unlike the rigid model, the compliant model considers a penetration of the contact point below the contact surface, when $p_{i}^{z}<0$. The graph of the rigid model, with the specific shape of a right angle, is called a Signorini graph.

Impacts. Since rigid contact models do not allow penetration of the contact points below the contact surfaces, when a point, $\boldsymbol{p}_{i}$, reaches a contact surface, $p_{i}^{z}=0$, with a velocity, $\dot{p}_{i}^{z}<0$, this velocity has to change instantaneously in order to satisfy the no-penetration assumption, $p_{i}^{z} \geq 0$ [26]. What happens in this situation is an impact, a discontinuity of the velocity, where we need to distinguish the velocity of the robot before impact, $\dot{\boldsymbol{q}}^{-}$, and its velocity after impact, $\dot{\boldsymbol{q}}^{+}$. A straightforward integration of the Lagrangian dynamics (48.2) over a time singleton [27] gives us a relation between pre- and post-impact velocities and impulsive forces $\boldsymbol{F}_{i}$ :

$$
\boldsymbol{M}(\boldsymbol{q})\left(\dot{\boldsymbol{q}}^{+}-\dot{\boldsymbol{q}}^{-}\right)=\sum_{i} \boldsymbol{C}_{i}(\boldsymbol{q})^{\top} \boldsymbol{F}_{i}
$$

But in order to completely define the impact law and compute the postimpact velocity, we also need a model of the impulsive forces. Unfortunately, this is a complex problem still open to research [28, 29], especially in situations of multiple contacts which are the standard situation for legged robots. The usual approach for legged robots is therefore to assume the post-impact behavior, usually considering that the contact points eventually stick to the contact surfaces:

$$
\dot{\boldsymbol{p}}_{i}^{+}=\boldsymbol{C}_{i} \dot{\boldsymbol{q}}^{+}=0
$$

This is, however, a strong assumption, and reality can be much more complex, as demonstrated in [30]. Moreover, a recent study shows that post-impact sticking may not always be desirable [31].

Impacts are central in most stability analyses of passive dynamic walking machines (see Section 48.6), but they can be potentially destructive for the 
mechanical parts of a robot. As a result, they are often carefully avoided and therefore generally disregarded outside of passive dynamic walkers. A recent study shows however that this is probably a mistake [32].

Hybrid and nonsmooth dynamics. With rigid contacts, the dynamics of legged robots appears to switch, depending on the contact situations (48.20) or (48.21). Discontinuities of the state of the robot also occur at impacts. A classical way to combine these different aspects is with a hybrid dynamical system. This approach has however some limitations [33, 34], the most obvious one being its incapacity to handle properly Zeno behaviors, infinite accumulations of impacts in finite time [35]. An example of such a situation is when a legged robot is rigidly standing on one foot, and gently rocking from one contact edge to the opposite contact edge, with a continuously decreasing period (at least according to the perfectly rigid model).

Impacts with multiple contacts and Zeno behaviors are not the only difficulties with rigid contact models: there is also the Painlevé paradox, tangential impacts, impacts without collisions, etc. The nonsmooth dynamics approach $[25,27,33,34]$, where the Lagrangian dynamics is turned into a measure differential equation, accelerations being abstract measures, velocities being functions with locally bounded variations, appears to be a much more adequate way to deal with all these intricacies. But it has not been widely adopted for legged robots because of a significant increase in mathematical complexity.

Many of the complexities of rigid body contact dynamics can be avoided by discretizing the system dynamics in time and reasoning about the integral of contact forces acting over a time step. In particular, no distinction is made between impulses and finite contact forces over a time step. By conservatively approximating the friction cone as a polyhedron, forward dynamics can be cast as a linear complementarity problem (LCP) [36], for which efficient solvers exist. This has become a popular formulation for simulation of rigid bodies in frictional contact and it has recently seen applications to control design for legged systems [37].

\subsection{Stability analysis, not falling down}

The robot model described above represents a complex, constrained, nonlinear dynamical system. Our goal for this section is to understand the long-term behavior of this dynamical system, to answer questions like, "Will the robot fall down?" As we will see in the following sections, this long-term behavior can be changed by open-loop or closed-loop feedback control, with the explicit goal of minimizing the likelihood that the robot will fall down.

For controlled nonlinear dynamical systems, there are a number of useful concepts relating to their safeness and stability, including:

- Fixed points. Stable fixed points represent the static postures in which the robot can safely stand still. 
- Limit cycles. Limit cycles provide a natural extension of fixed-point analysis to periodic walking or running motions.

- Viability. Viability is a concept of controlled invariance, which analyzes the set of states from which the robot is able to avoid to fall. Unfortunately, this property can be intractable to compute.

- Controllability. Controllability provides a slightly restricted notion of viability, analyzing the set of states from which the robot is capable of returning to a particular fixed point (or limit cycle). This can be more tractable to compute than viability, especially for simple models.

In addition, if there are unknown errors in the robot model, if there is uncertainty about the environment (e.g., the location of the ground), or if there are unmodeled disturbances, then additional tools are available from robustness analysis and stochastic stability:

- Robust stability. Robust stability (or viability) examines the properties of the system considering worst-case (bounded) disturbances. For instance, a robust controller may be able to guarantee that a fixed point is stable even if the estimate of the mass of the trunk is wrong by $\pm 10 \%$.

- Stochastic stability. Stochastic analysis provides tools to investigate the probability of falling down. For many robot disturbance models, the system will always fall eventually (with probability one), but analysis can reveal long-living "metastable" distributions.

- Input-output stability. This analysis treats a particular disturbance as an input, a performance criteria as output, and attempts to compute a relative gain or sensitivity of the robot performance due to this input.

- Stability margins. Robustness analysis can be difficult. In practice control designers often settle for the system staying comfortably away from the boundaries of deterministic stability.

We give a very brief overview of these tools in the remainder of this section, with an attempt to emphasize features of the analysis that are particular to legged robots.

\subsubsection{Fixed points}

Given a first-order ordinary differential equation governed by $\dot{\boldsymbol{x}}=\boldsymbol{a}(\boldsymbol{x})$, a fixed point of the system is a state, $\boldsymbol{x}^{*}$, for which $\boldsymbol{a}\left(\boldsymbol{x}^{*}\right)=0$. The governing equations for a legged robot, given in Section 48.2, are second-order, take a control input, and potentially require a differential inclusion to describe the contact forces, but the notion of a fixed point is still meaningful. Here a fixed point is a configuration, $\boldsymbol{q}^{*}$, for which there exists feasible control inputs, $\boldsymbol{u}^{*}$, and feasible contact forces, $\boldsymbol{f}^{*}$, so that $\dot{\boldsymbol{q}}^{*}=\ddot{\boldsymbol{q}}^{*}=\mathbf{0}$ is a solution to equation (48.2). A fixed point of this system is a posture in which the legged robot is able to stand still. 


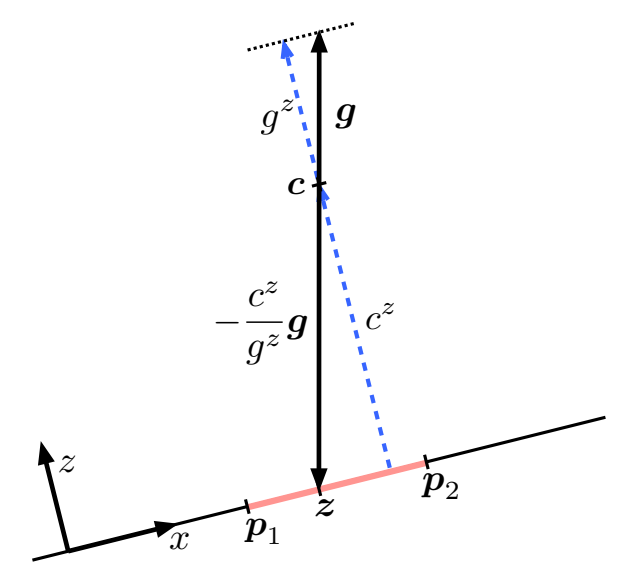

Figure 48.5: A necessary static equilibrium condition on a flat ground is that the $\mathrm{CoM}, \boldsymbol{c}$, must project on the ground along the gravity vector, $-\boldsymbol{g}$, inside the convex hull of the contact points, $\boldsymbol{p}_{i}$, also known as the support polygon (in red).

In static situations, when $\ddot{\boldsymbol{c}}=\dot{\boldsymbol{L}}=\mathbf{0}$, the ODI (48.13) gives the following necessary condition

$$
\boldsymbol{c}^{x, y}-\frac{c^{z}}{g^{z}} \boldsymbol{g}^{x, y}=\boldsymbol{z}^{x, y} \in \operatorname{conv}\left\{\boldsymbol{p}_{i}^{x, y}\right\}
$$

for a static equilibrium on a flat ground. This necessary condition states that the CoM, $\boldsymbol{c}$, must project on the ground along the gravity vector, $-\boldsymbol{g}$, inside the convex hull of the contact points, $\boldsymbol{p}_{i}$, also known as the support polygon (Figure 48.5). This is a very well known and widely used necessary condition. Note, however, that it is valid only when considering contacts with a flat ground [15].

Once a fixed point has been found, one would often like to examine its stability. For smooth nonlinear differential equations, local stability can often be established via linearizing the dynamics at the fixed point and then applying well-known tools from linear systems theory. Indeed, these tools can also be applied here, under the strong assumption that the contact conditions do not change (e.g., either $f_{i}^{z}=0$ and $p_{i}^{z} \geq 0$, or $f_{i}^{z} \geq 0$ and $p_{i}^{z}=0$ ). Each active contact adds an equality constraint to the linearized system and the eigenvalues of this constrained linear system are only meaningful in the minimal coordinates; e.g., for a robot standing on horizontal ground the assumption precludes the ability to examine a perturbation of the entire robot in the vertical direction. Note that in a hybrid model of the legged robot, this assumption is equivalent 


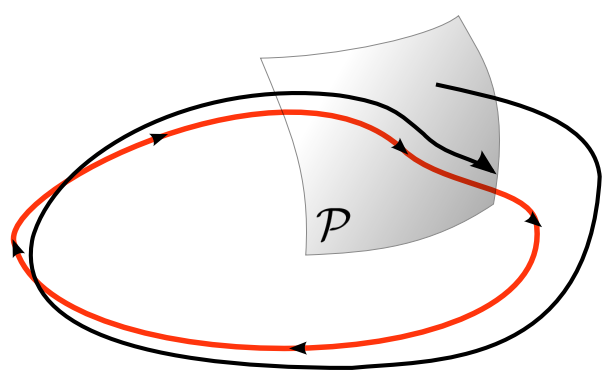

Figure 48.6: Illustration of a Poincaré surface, $\mathcal{P}$, defined on a periodic orbit (red) and a trajectory converging to the orbit (black).

to linearizing within a single hybrid mode, and it is common practice to describe each hybrid mode in its minimal coordinates [38]. Once local stability is established, it may also be possible to understand the region of attraction of the fixed point $[39,40])$. Evaluating the stability of these models through changes in contact configurations is a new and important area of research. For recent work, see for instance [41].

\subsubsection{Limit Cycles}

A natural extension of fixed-point analysis to walking and running motions is to examine the existence and stability of periodic orbits, or limit cycles, of the dynamical system. Here a periodic orbit is a solution $\{\langle\boldsymbol{q}(t), \boldsymbol{u}(t), \boldsymbol{f}(t)\rangle \mid t \in[0, \infty)\}$ of the dynamical system with a finite period $T>0$ such that $\boldsymbol{q}(t+T)=$ $\boldsymbol{q}(t), \boldsymbol{u}(t+T)=\boldsymbol{u}(t)$, and $\boldsymbol{f}(t+T)=\boldsymbol{f}(t)$. Almost always, periodic orbits are discovered numerically using techniques from motion planning like those described in Section 48.4. For passive, or very minimally actuated robots, the very existence of a periodic solution can be exciting [42]; robots with more actuators typically have an abundance of periodic solutions and planning techniques can be used to find solutions that, for instance, minimize the a quantity of interest such as the cost of transport [43] or a measure of open-loop stability [44].

A periodic solution, $\left\langle\boldsymbol{q}_{0}(\cdot), \boldsymbol{u}_{0}(\cdot), \boldsymbol{f}_{0}(\cdot)\right\rangle$, with period $T$, is (asymptotically) orbitally stable [45] or limit-cycle stable if, given initial conditions, $\boldsymbol{q}(0)$, we have

$$
\lim _{t \rightarrow \infty}\left[\min _{0 \leq t^{\prime}<T}\left\|\boldsymbol{q}(t)-\boldsymbol{q}_{0}\left(t^{\prime}\right)\right\|\right]=0 .
$$

The key feature here that there is no requirement that system trajectories converge in time; only the distance to the closest point on the orbit must go to zero. This stability can be defined for initial conditions restricted to a local neighborhood of $\boldsymbol{q}_{0}(\cdot)$, for a region containing $\boldsymbol{q}_{0}(\cdot)$, or globally (though no interesting legged robot is globally stable) and for inputs determined by a variety of open- or closed-loop control policies, $\boldsymbol{u}(t)=\boldsymbol{\pi}(t, \boldsymbol{q}(t), \dot{\boldsymbol{q}}(t), \boldsymbol{f}(t))$. Under mild 
conditions, a sufficient criteria for establishing orbital stability is establishing fixed-point stability of a Poincaré map [46], as illustrated in Figure 48.6. Due to the limitation that finding periodic solutions is typically done numerically, Poincaré map analysis is also typically done numerically, e.g., using finite differences to evaluate a local linearization of the map. This does not necessarily preclude rigorous analysis, and techniques exist to compute regions of attraction to hybrid limit cycles despite potential numerical inaccuracies [47]. Finally we note that limit cycle analysis can be applied (carefully) to solutions that are not periodic in all states - for instance, the $x, y$ location of the floating base should be left out if the robot needs to make forward progress!

The primary advantage of limit cycle analysis is that, using the Poincaré map and related methods, many of the tools from fixed point analysis and linear systems theory can be easily extended to evaluate (local) stability and even to design a (locally) stabilizing controller. However, this is a very limited definition of locomotion; useful locomotion through non-trivial environments will likely require aperiodic motions.

Extensions to aperiodic stability. The notion of stability requires convergence to some nominal solution as time goes to infinity, thus the nominal solution must be defined over an infinite interval. A common approach involves stitching together periodic solutions with provably bounded finite-time transition maneuvers which can switch between them [48]. Orbital stability of an (infinite) aperiodic trajectory can be established using a transverse linearization or "moving Poincaré section" [49]. For real-world situations where the entire desired trajectory cannot be known apriori, these guarantees can also be provided using online motion planning and receding-horizon control [50].

\subsubsection{Viability}

If the objective of a legged robot is simply to avoid falling down, it can be possible then to directly define (at least theoretically) the set of postures, $\mathcal{F} \subseteq$ $\mathbb{R}^{N+6}$, where the robot has fallen down and that the robot should simply avoid. This leads to introducing the set of states from which the robot is able to avoid entering $\mathcal{F}$ - the set of states from which the robot is able to avoid falling down. These states will be called viable, following the viability theory developed in [51] for general ODIs and introduced in the analysis of walking robots in [52]. In principle, the set of all viable states represents a safe operating envelope for the robot. It is typically intractable to compute explicitly but it can be approached indirectly in the case of walking robots in the following way.

Center of mass dynamics during horizontal walking. In case the robot is walking on a horizontal ground, the $z$ axis is aligned with gravity, so $\boldsymbol{g}^{x, y}=0$ and the ODI (48.13) becomes

$$
\boldsymbol{c}^{x, y}-\frac{c^{z}}{\ddot{c}^{z}+g^{z}} \ddot{\boldsymbol{c}}^{x, y}+\frac{1}{m\left(\ddot{c}^{z}+g^{z}\right)} \boldsymbol{S} \dot{\boldsymbol{L}}^{x, y} \in \operatorname{conv}\left\{\boldsymbol{p}_{i}^{x, y}\right\} .
$$


We can observe that it is linear with respect to the horizontal motion $\boldsymbol{c}^{x, y}, \ddot{\boldsymbol{c}}^{x, y}$ of the CoM and variations of the angular momentum $\boldsymbol{L}^{x, y}$. The vertical motion $c^{z}, \ddot{c}^{z}$ of the CoM can usually be bounded, but in order to simplify the following derivations, let us suppose that the CoM moves strictly horizontally above the ground, i.e. $c^{z}$ is constant and $\ddot{c}^{z}=0$, so the ODI becomes:

$$
\boldsymbol{c}^{x, y}-\frac{c^{z}}{g^{z}} \ddot{\boldsymbol{c}}^{x, y}+\frac{\boldsymbol{S} \dot{\boldsymbol{L}}^{x, y}}{m g^{z}} \in \operatorname{conv}\left\{\boldsymbol{p}_{i}^{x, y}\right\} .
$$

Variations of the angular momentum $\boldsymbol{L}$ can also be bounded in the $x$ and $y$ directions, but to make things as simple as possible, let us consider these variations to be equal to 0 , so the ODI takes a very simple second order linear form:

$$
\boldsymbol{c}^{x, y}-\frac{c^{z}}{g^{z}} \ddot{\boldsymbol{c}}^{x, y}=\boldsymbol{z}^{x, y} \in \operatorname{conv}\left\{\boldsymbol{p}_{i}^{x, y}\right\}
$$

An example of inevitable fall. Let us consider furthermore a situation where, for some reason (e.g., a cliff), no contact with the ground can be realized beyond a certain line (Figure 48.7), with $\boldsymbol{a}$ a vector orthogonal to this line and pointing beyond it. If the CoM of the robot reaches this line at a time $t_{0}$ with a speed pointing beyond it $\left(\boldsymbol{a}^{\top} \dot{\boldsymbol{c}}^{x, y}\left(t_{0}\right)>0\right)$, this linear ODI can be integrated analytically very simply and lead to the following inequality [53], valid at all time $t \geq t_{0}$ :

$$
\boldsymbol{a}^{\top}\left(\boldsymbol{c}^{x, y}(t)-\boldsymbol{c}^{x, y}\left(t_{0}\right)\right) \geq \frac{\boldsymbol{a}^{\top} \dot{\boldsymbol{c}}^{x, y}\left(t_{0}\right)}{\omega} \sinh \left(\omega\left(t-t_{0}\right)\right)
$$

with

$$
\omega=\sqrt{\frac{g^{z}}{c^{z}}}
$$

The right hand side is increasing exponentially with time. As a result, the position $\boldsymbol{c}^{x, y}$ of the CoM is diverging exponentially in the direction of the vector $\boldsymbol{a}$, leading inexorably to a fall.

A sufficient condition for viability. In the simplified linear case developed above, the inequality (48.30) establishes that in the case of a fall, the motion of the CoM diverges exponentially, so the integral of the norm of any $n^{\text {th }}$ derivative of its position,

$$
\int_{t_{0}}^{\infty}\left\|\boldsymbol{c}^{(n)}(t)\right\| d t
$$

would be infinite. Therefore, if we can find a finite value for such an integral from a given initial state of the robot, we can conclude that this state is viable.

\subsubsection{Controllability}

For any dynamical system, an initial condition, $\boldsymbol{x}_{0}$, is controllable to a final state, $\boldsymbol{x}_{f}$, if there exists a feasible input trajectory, $\boldsymbol{u}(\cdot)$, which takes the system 


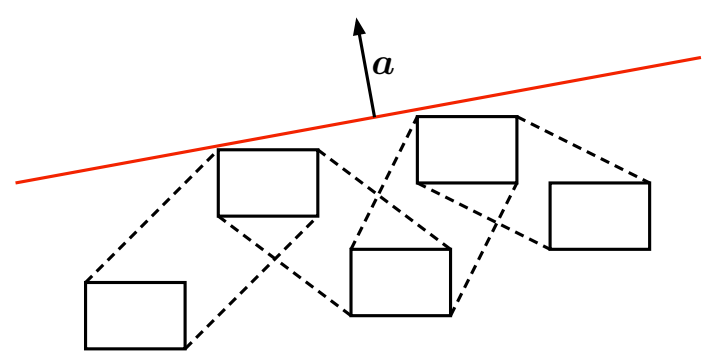

Figure 48.7: A situation where, for some reason, no steps can be undertaken beyond a certain line (in red), with $\boldsymbol{a}$ a vector orthogonal to this line and pointing beyond it.

from $\boldsymbol{x}_{0}$ to $\boldsymbol{x}_{f}$. An initial state, $\boldsymbol{x}_{0}$, is denoted finite-time controllable to $\boldsymbol{x}_{f}$ if the minimum time required is finite. Controllability analysis is a well understood topic for linear dynamical systems, where a simple rank condition on the "controllability matrix" provides necessary and sufficient conditions to establish whether or not any initial condition can be driven to any final state [54]. For nonlinear systems, the property is dependent on both the initial and final states.

In the case of legged robots, instead of considering the set of all viable states introduced earlier, we could consider instead the more limited set of states that are controllable to stable fixed-points - states that can come to a stable stop after a given number of steps. This is a sufficient condition for viability. Such states have been called capturable [55], and arguably encompass most of the states of interest for legged robots. A variant would be to consider states from which the robot is able to reach a stable limit cycle, or any other state which is known to be viable in the first hand.

The Capture Point. In the simple linear case developed above, the capturable states can be identified analytically with the help of the compound variable

$$
\xi=c+\frac{1}{\omega} \dot{c}
$$

introduced independently as the eXtrapolated Center of Mass (XCoM) [56], the Capture Point [57] or the divergent component of the dynamics [58]. These three denominations correspond to three key properties of this variable. First of all, a trivial reformulation gives

$$
\dot{\boldsymbol{c}}=\omega(\boldsymbol{\xi}-\boldsymbol{c})
$$

revealing that this point $\boldsymbol{\xi}$ is the point where the CoM is converging to, hence the extrapolated CoM . Following the linear dynamics (48.29), the horizontal 


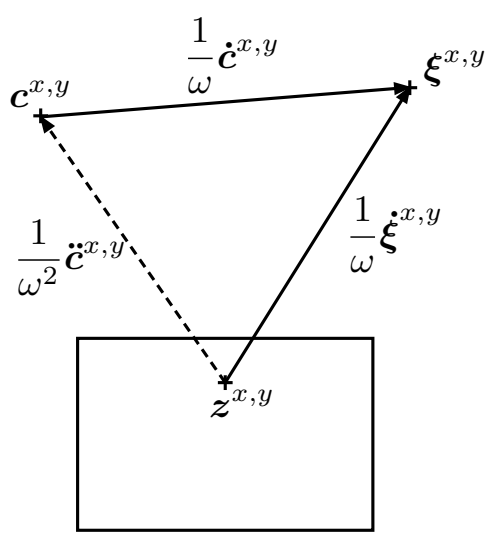

Figure 48.8: The speed of the CoM, $\boldsymbol{c}$, points towards the Capture Point, $\boldsymbol{\xi}$, while the speed of this Capture Point points away from the CoP, $\boldsymbol{z}$. The combination of these two first-order dynamics gives the acceleration, $\ddot{\boldsymbol{c}}$, pushing the CoM away from the CoP.

motion of this point satisfies

$$
\dot{\boldsymbol{\xi}}^{x, y}=\omega\left(\boldsymbol{\xi}^{x, y}-\boldsymbol{z}^{x, y}\right),
$$

where we can see that this point $\boldsymbol{\xi}$ diverges away from the $\mathrm{CoP} \boldsymbol{z}$. But if this point is above the support polygon, if we have

$$
\boldsymbol{\xi}^{x, y} \in \operatorname{conv}\left\{\boldsymbol{p}_{i}^{x, y}\right\},
$$

we can have $\boldsymbol{z}^{x, y}=\boldsymbol{\xi}^{x, y}$ so the point $\boldsymbol{\xi}$ can stay motionless, and the CoM will converge to it and come to a stop, hence it can be seen as a Capture Point (CP). Finally, we can observe that the second-order linear dynamics (48.29) has been decomposed here as two first-order linear dynamics (48.34)-(48.35), the first one being stable, with the CoM converging to the XCoM, the second one being unstable, with the $\mathrm{XCoM}$ diverging away from the CoP, hence the divergent part of the dynamics (48.29). Here appears an interesting structure relating the motion of the points $\boldsymbol{z}, \dot{\boldsymbol{c}}$ and $\boldsymbol{\xi}$, shown in Figure $48.8[59,60]$.

An important observation here is that if the state of the robot satisfies the condition (48.36), then the robot can stop without making any step and it is capturable and viable. Further analysis, considering any given number of steps and a non-zero angular momentum, $\boldsymbol{L}$, can be found in [60].

\subsubsection{Robust or stochastic stability}

For the deterministic robot dynamics models described in Section 48.2, the concepts of fixed-points, limit cycles, viability, and controllability form the core 
issues of analysis. However, in the presence of disturbances, such as model inaccuracies or unpredicted variation in terrain, tools from robust verification must be called upon to characterize the stability of a system. Broadly speaking, robust stability analysis exists in two forms. In worst-case analysis, an upper bound is given for a disturbance and the goal is to demonstrate that for any disturbance within this bound, the system will not reach an unstable state. Alternatively, stochastic analysis can be performed where a disturbance probability distribution is specified with the goal being to demonstrate that the system avoids unstable states with high probability. Worst-case analysis has received surprisingly little attention to date in the legged robotics community, however analysis approaches based on Lyapunov functions [38, 47, 41] can be easily extended using the notion of a common Lyapunov function [61]. One challenge here is that the nominal solutions, e.g. the exact shape of the nominal limit cycle, are often parameter dependent: the system might still be stable with different parameters, but to a slightly different limit cycle.

Stochastic stability has received relatively more attention in the legged robotics community, perhaps because many interesting models of possible disturbances do result in robots that walk for a long time, but do eventually fall over. Such systems cannot be classified as stable, but it seems incomplete to simply call them unstable. Such systems are said to be metastable, a concept first established in the physics community [62] and later used to analyze walking systems [63]. From this point of view, the stability of a system can be characterized by its mean time to failure.

Recall the idea of Poincaré maps described above, but now consider the discrete time stochastic return map dynamics for a periodic walking system,

$$
\boldsymbol{x}_{k+1}=r\left(\boldsymbol{x}_{k}, h_{k}\right),
$$

where $h_{k} \sim P(h)$ is a random variable that, e.g., captures the terrain height at time step $k$. Discretizing the state space $\mathcal{X} \subseteq \mathbb{R}^{(N+6)} \times \mathbb{R}^{(N+6)}$ into $d$ states allows us to define the Markov process based on the above return map:

$$
\boldsymbol{p}_{k+1}=\boldsymbol{T} \boldsymbol{p}_{k},
$$

where $p_{k} \in \mathbb{R}^{d}$ is the state probably distribution vector and $\boldsymbol{T}$ is a stochastic matrix with entries $T_{i j}=\operatorname{Pr}\left(\boldsymbol{x}_{k+1}^{i} \mid \boldsymbol{x}_{k}^{j}\right)$. If we assume that all failure states are absorbing and can be grouped together into a single state, this corresponds to a column in $\boldsymbol{T}$ containing a single 1 and $d-1$ zeros. The largest eigenvalue of $\boldsymbol{T}$ must then be $\lambda_{1}=1$ with a corresponding eigenvector, $\boldsymbol{v}_{1}$, describing the stationary distribution of the absorbing state. Interestingly, the second largest eigenvalue has been found to be close to unity, $\lambda_{2} \approx 1$, and much larger than the remaining $d-2$ eigenvalues, for several simple walking systems [63]. This suggests the existence of a metastable neighborhood in state space captured by the distribution $\boldsymbol{v}_{2}$ with an associated time constant $\tau=-1 / \log \left(\lambda_{2}\right)$. Other approaches to computing these stochastic stability bounds are also possible [64]. 


\subsubsection{Input-output stability}

Input-output methods define sensitivity measures to summarize the system's response to disturbances. For example, for a walking system, one could define a vector $\boldsymbol{l} \in \mathbb{R}^{m}$ composed of $m$ gait variables that directly relate to failure modes (such as foot-ground clearance) and measure the effect that a set of input disturbances, $\boldsymbol{w}$, have on their values. The gait sensitivity norm does exactly this [65]; here the dynamic system response $\|\partial \boldsymbol{l} / \partial \boldsymbol{w}\|_{2}$ is used as a measure of disturbance rejection. How is this response actually computed? In practice, computing the system response can be done experimentally by generating a variety of perturbations and measuring the effect in the gait variables. Although laborious, this approach is probably the most appropriate for physical systems. Alternatively, for simulated limit cycle walkers, linearization of the Poincaré return map and perturbation analysis yields a complete characterization of the dynamic system response.

More general tools from nonlinear control theory can also be applied. The $L_{2}$ gain is a sensitivity measure that has seen widespread application in nonlinear control theory. For complex dynamical systems such as walking robots, an upper bound on the $L_{2}$ gain can be approximated using storage functions, which can often be computed using convex optimization [66, 67].

\subsubsection{Stability margins}

In practice, computing performance measures like the ones mentioned above for complex walking systems can be very difficult. As a result, researchers often employ heuristic stability margins to keep the robot away from the boundary of stability. Here the definition of stability can vary widely. The simplest stability margins rely only on the static configuration of the robot, where if the CoM ground projection leaves the support polygon, this corresponds to an uncompensated moment on the foot resulting in a rotation along its edge. Popular static metrics include the distance between the ground projection of the CoM and the edges of the support polygon or the minimum potential energy required to tip the robot over sideways [68]. Because these stability criteria are based only on statics, they are only practically useful for slow (or "quasi-static") motions.

The most commonly used dynamic stability margin requires that the CoP (or ZMP) remains within the boundary of the support polygon. When this condition is satisfied, the foot cannot rotate around the boundary of the support polygon. Note that this is certainly very conservative - many bipedal robots walk dynamically even with point feet (resulting in a CoP that is always on the boundary of the support polygon) - but this metric has proven extremely useful for flat-foot dynamic walking. Goswami introduced the related concept of the foot rotation indicator (FRI) [69] that is defined as the point on the ground where the net ground reaction force would have to act to keep the foot from rotating; this point need not stay inside the support polygon and can therefore be applied for a wider range of gaits. However, the stability claims based on 
these criteria are valid only on flat terrain and they do not constitute necessary or sufficient conditions for stable walking in general [15, 55].

When the CoM projection exits the support polygon, there are two ways to make it return: by accelerating the CoM in the direction of the support polygon through changes in angular momentum or by changing the support polygon by taking a step. This basic insight led to stability margins based on capture regions $[55,60]$. A capture region is simply the set of all capture points (defined in Section 48.3.4). The capture margin is a metric that determines the "capturability" of a walking system. If the capture region and support polygon overlap, it is defined as the maximum distance between the points in the capture region and the closest edge of the support polygon, otherwise it is the negative distance between the capture region and support polygon. Note that large values under this metric are related to the total capture region area, which is in turn related to the ability of the walking system to stabilize itself, e.g., with upper body motions that affect angular momentum [60]. Extensions to $n$-step capture margins have also been developed and described in detail [55].

\subsection{Generation of dynamic walking and run- ning motions}

As mentioned in the brief historical introduction to this Chapter, early legged robots were maintaining quasi-static equilibrium postures at all time. The transition to more dynamic motions took place at the beginning of the 1980s, associated with biped [4] and monopod [5] robots. As a result, this Section on dynamic legged locomotion will focus on results obtained mostly with biped robots, although most of the methods discussed here could be applied as well to other legged robots.

\subsubsection{Early offline motion generation schemes}

The very limited computing power of early robots allowed very limited online decisions. As a result, trajectories were usually computed offline, especially in complex situations such as for legged robots. Online motion generation has finally become possible only by the year 2000, thanks to the exponential increase in computing power and the continuous progress of numerical methods during the last decades.

Trajectory optimization. One of the earliest approaches to computing walking and running motions was through numerical optimization [70]. The idea is to leverage the capacity of trajectory optimization methods to take into account nonlinear dynamics and objectives such as minimizing energy consumption, and compute the corresponding optimal motion. Unfortunately, the dynamics of legged robots are so complex that this approach was still stuck with simple planar models by the end of the 1990s [71, 72, 73, 74, 75]. It is only by the year 
2000 that the first optimal walking motions for complete 3D models could be computed [76, 77].

Fortunately, numerical methods have continued to improve (as discussed in 48.6.1), allowing researchers to solve complex problems such as optimizing trajectories and the mass distribution of the robot simultaneously and maximizing the open-loop stability of trajectories [78].

Unfortunately, this approach still requires time consuming computations that often cannot be realized online. Not being able to compute walking or running motions online limits the robots to a predefined set of precomputed actions, potentially ruining their versatility and reactivity. One way to alleviate this serious limitation is to generate a database of trajectories [79] that can be queried online, possibly conditioned on orders given to the robot [80] or the current state of the robot in order to improve its stability [81, 82, 40].

Artificial synergy synthesis and the ZMP approach. Partitioning the problem is another early approach, that aimed at palliating the complexity of the dynamics of legged robots that hindered trajectory optimization. The idea is to assign some degrees of freedom of the robot to take care of dynamic constraints such as the ODI (48.13), allowing the rest of the robot to be operated more or less independently. This general approach has been called artificial synergy synthesis [10].

The original proposition in [10] was to use trunk rotations to ensure dynamic feasibility while the legs of the robot executed a given pre-recorded motion. More precisely, leg motions and contact points, $\boldsymbol{p}_{i}$, being predefined, the trajectory of the $\mathrm{CoP}, \boldsymbol{z}$, could be predefined accordingly, so that it was just a matter of solving the ODE (48.13) for this predefined $\boldsymbol{z}$ to obtain the required rotations of the trunk. This original proposition was demonstrated experimentally 20 years later on the Waseda University WL-12RV biped walking robot [83]. This method of predefining the trajectory of the CoP has eventually been called the ZMP approach to walking motion generation (remember the ZMP is just another name given to the $\mathrm{CoP}$ ). This ZMP approach has been associated later with having feet always flat on the ground, excluding heel and toe rotation phases. Note, however, that such phases were considered in the original proposition [10].

Some aspects of this proposition can be questioned. It has been argued first of all that predefining the evolution of the $\mathrm{CoP}$ is not necessary nor even desirable $[84,85]$. Then, the ODI (48.13) clearly shows that dynamic feasibility depends on both variations of the angular momentum, $\boldsymbol{L}$, and motion of the $\mathrm{CoM}, \boldsymbol{c}$, with respect to contact points, $\boldsymbol{p}_{i}$. While trunk rotations mostly involve variations of the angular momentum, a recent analysis showed that this has only a weak influence on the balance of legged robots [86]. Dynamic feasibility can be handled much more efficiently by adapting the motion of the CoM, which has been the prevailing approach.

The core idea of partitioning the motion of the robot builds on a profound and far-reaching observation: if the robot has a sufficient number of degrees of 
freedom and sufficient control authority, then every part of the motion that is not involved in the dynamic constraints - everything but angular momentum and motion of the CoM with respect to contact points - can be operated more or less independently, and appears therefore to be relatively peripheral to the problem of legged locomotion. It is the same key observation that implicitly drives the Templates and Anchors approach and the long history of simple biomechanical models of legged locomotion that focus on a few meaningful degrees of freedom, mostly the motion of the CoM with respect to contact points, and abstract all the rest $[87,88,89]$. This idea has been tremendously successful in the legged locomotion research community.

\subsubsection{Online motion generation: a Model Predictive Con- trol point of view}

If the algorithms for motion generation could work sufficiently fast to be applied online, then the robot could achieve reactivity and robustness by continuously adapting the motions to the current state of the robot and its environment. But therein lies a problem: how can we make sure that the continuous re-evaluation of online decisions maintains long term viability? One solution comes from the Model Predictive Control (MPC) theory.

The online motion generation schemes that allow most of the great humanoid robots of today and yesterday to walk and run can all be related to MPC theory and can all be seen as variants of the same MPC scheme, although they have rarely been introduced in that way. Most adopt the artificial synergy synthesis approach described in the previous Section, focusing almost exclusively on the motion of the CoM with respect to contact points and assuming that the rest of the robot can be operated more or less independently.

Viability and capturability, Optimal Control and Model Predictive Control When trying to identify viable states in Section 48.3.3, the first option was to check if the integral (48.32) could have a finite value. A classical result from optimal control theory is that an optimal control law minimizing this integral would make it decrease with time. As a result, if this integral was finite in the beginning, it would stay finite: if the state of the robot was viable in the beginning, it would stay viable. The problem is, the dynamics of legged robots is too complex to be able to compute such an optimal control law in the general case.

Model Predictive Control is one way to obtain a computable approximation to this optimal control law. The fundamental idea is to introduce a terminal constraint [90], imposing that the integral (48.32) is equal to zero after a finite length of time. Introducing such an artificial constraint naturally yields a suboptimal control law, but this allows considering the integral (48.32) over only a finite length of time while preserving viability. Note that enforcing viability in finite time means in this case enforcing capturability: here, the terminal constraint is a capturability constraint. 
Many MPC variants have been proposed [91], but two extremes are going to be significant in the following. One extreme is to observe that minimizing an integral of the form (48.32) is not necessary to obtain a viable behavior: the terminal, capturability constraint can be sufficient in this respect [92]. In this case, however, it is not possible to continuously re-evaluate and adapt the motion to the state of the system: this must be done at carefully chosen moments, what limited the reactivity of the robots. The other extreme is to observe that the terminal constraint is not absolutely necessary [93]: simply minimizing a truncated version of the integral (48.32) over a sufficiently long but finite length of time can still lead to an integral decreasing with time, ensuring viability. In this case, it is possible to continuously re-evaluate and adapt the motion to the state of the system without any problem.

Let us see now how all this theory is applied to the online generation of walking and running motions. In all the following examples, the robot is supposed to walk on a flat horizontal ground.

Predefined footsteps, and capturability constraint. Let us have a look first at motion generation schemes implementing the original ZMP approach, with predefined footsteps and a predefined $\mathrm{CoP}$, and considering a capturability constraint to ensure viability. Let us begin with the walking motion generation scheme implemented in the long series of Waseda University humanoid robots [94], which considers a four point mass model with predefined motion except for the horizontal motion of the waist and trunk masses which is assigned to follow a reference trajectory for the CoP. An iterative procedure based on Fast Fourier Transforms is used then to solve the dynamics

$$
\frac{\sum m_{i}\left(\ddot{c}_{i}^{z}+g^{z}\right) \boldsymbol{c}_{i}^{x, y}-m_{i} c_{i}^{z} \ddot{\boldsymbol{c}}_{i}^{x, y}}{\sum m_{i}\left(\ddot{c}_{i}^{z}+g^{z}\right)} \longrightarrow \boldsymbol{z}_{\text {ref }}^{x, y}
$$

In order to execute this scheme online, a capturability constraint is introduced, imposing that the robot is always able to stop within two steps [95]. Unfortunately, details about the choice of the reference $\mathrm{CoP}$ and the exact terminal constraint are not disclosed.

The walking motion generation scheme implemented in the Munich University Johnny robot [96] considers only a three point mass model with predefined motion, except for the horizontal motion of the main mass in the trunk which is assigned to follow a piecewise linear reference trajectory for the CoP. One degree of freedom is left in this reference trajectory in order to impose a terminal constraint on the position of the CoM at the end of the next two steps:

$$
c^{x, y}=c_{r e f}^{x, y} .
$$

This constraint appears to be incomplete in imposing capturability, what would require to consider also the velocity of the CoM (see Section 48.3.3).

The walking motion generation scheme implemented in the Honda Asimo robot [58] is very similar, three point masses and a piecewise linear reference for the $\mathrm{CoP}$ with one degree of freedom left to satisfy the terminal constraint. The 
difference lies in the terminal constraint which is a true capturability constraint, imposing cyclicity of the motion through the Capture Point/XCoM/divergent component of the dynamics at the end of the next step:

$$
\boldsymbol{\xi}^{x, y}=\boldsymbol{\xi}_{r e f}^{x, y} .
$$

The walking motion generation scheme implemented in the Tokyo University H7 robot [97] considers the whole dynamics (48.13) of the robot. The whole motion of the robot is predefined, except the horizontal motion of the CoM which is assigned to follow a reference trajectory for the CoP, set in the middle of the contact points. An iterative procedure is used then to solve the dynamics

$$
\boldsymbol{c}^{x, y}-\frac{m c^{z} \ddot{\boldsymbol{c}}^{x, y}-\boldsymbol{S} \dot{\boldsymbol{L}}^{x, y}}{m\left(\ddot{c}^{z}+g^{z}\right)} \longrightarrow \overline{\boldsymbol{p}_{i}^{x, y}} .
$$

The terminal constraint (48.40) on the position of the CoM (incomplete with respect to capturability) is also considered at the end of the next two steps.

The walking and running motion generation scheme implemented in the Toyota Partner robot [98] is exactly the same, except for the terminal constraint which is a true capturability constraint imposing cyclicity of the motion through both the position and velocity of the CoM.

The walking and running motion generation scheme implemented in the Sony QRIO robot [99] follows a similar design, but considers only a single point mass at a constant height, with its horizontal motion assigned to minimizing the deviation of the CoP from the middle of the contact points:

$$
\min \int\left\|\boldsymbol{c}^{x, y}-\frac{c^{z}}{g^{z}} \ddot{\boldsymbol{c}}^{x, y}-\overline{\boldsymbol{p}_{i}^{x, y}}\right\|^{2} d t
$$

while imposing a capturability constraint on both the position and velocity of the CoM.

Another variant tested on the Kawada HRP-2 robot [100] also considers a single point mass at a constant height, but the $\mathrm{CoP}$ follows a piecewise polynomial trajectory:

$$
\boldsymbol{c}^{x, y}-\frac{c^{z}}{g^{z}} \ddot{\boldsymbol{c}}^{x, y}=\boldsymbol{z}_{\text {ref }}^{x, y}
$$

with some degrees of freedom left to satisfy the same capturability constraint as before, through both the position and velocity of the CoM. An important characteristic of this scheme is that the piecewise polynomial trajectory of the CoP may fluctuate strongly, threatening to violate the ODI (48.13). An automatic adjustment of the step timings is proposed therefore in order to minimize this risk.

All of these walking and running motion generation schemes try to impose capturability through terminal constraints, but some of them appear to fail properly doing so by only constraining the position of the CoM. None of them consider an integral of the form (48.32): the integral (48.43) does not match. We have seen earlier that in this case, it is possible to re-evaluate and adapt 
the motion at specific instants, what has been done when having to realize new steps, or when changing the walking speed or direction. But to adapt the motion to a perturbation, a specific observer would be required to trigger the adaptation at the correct instant [92], and it appears that this option hasn't been investigated: no state feedback has been experimented with these motion generation schemes.

Predefined footsteps, without capturability constraint. In the standard walking motion generation scheme implemented in the Kawada HRP-2 humanoid robot [101], the whole motion of the robot is predefined as before, except the horizontal motion of the CoM which is assigned this time to minimize the weighted integral

$$
\min \int\left\|\dddot{\boldsymbol{c}}^{x, y}\right\|^{2}+\beta\left\|\boldsymbol{c}^{x, y}-\frac{c^{z}}{g^{z}} \ddot{\boldsymbol{c}}^{x, y}+\frac{\boldsymbol{S} \dot{\boldsymbol{L}}^{x, y}}{m g^{z}}-\overline{\boldsymbol{p}_{i}^{x, y}}\right\|^{2} d t
$$

of the norm of the third derivative of the motion of the CoM and the deviation of the $\mathrm{CoP}$ from a reference in the middle of the contact points. This is clearly an integral of the form (48.32), and we have seen that viability can be ensured in this case if we consider a truncated version of this integral over a sufficiently long but finite length of time, typically the next two steps, without the need to impose any terminal constraint. We have also seen that it is possible in this case to continuously re-evaluate and adapt the motion to the state of the system, a clear improvement over the previous approaches based solely on terminal constraints. This has been validated experimentally in various situations, effectively adapting the walking motion to perturbations [102].

An interesting variant [103] introduces variations of the angular momentum, $\boldsymbol{L}$, as an additional variable to minimize the combined integral

$$
\min \int\left\|\dddot{\boldsymbol{c}}^{x, y}\right\|^{2}+\beta\left\|\boldsymbol{c}^{x, y}-\frac{c^{z}}{g^{z}} \ddot{\boldsymbol{c}}^{x, y}+\frac{\boldsymbol{S} \dot{\boldsymbol{L}}^{x, y}}{m g^{z}}-\overline{\boldsymbol{p}_{i}^{x, y}}\right\|^{2}+\gamma\left\|\boldsymbol{L}^{x, y}\right\|^{2} d t
$$

showing an improvement in the tracking of the generated motion (and the same closed loop robustness to small perturbations).

A problem however with these approaches is that simply minimizing the deviation of the $\mathrm{CoP}$ from the middle of the contact points doesn't preclude it from fluctuating, so the ODI (48.13) could be violated, especially in case of perturbations. This is monitored in [102] to trigger a change of footstep if necessary, but without any clear guarantee that this change is appropriate. For this reason, it has been proposed in [85] to impose the ODI (48.13) as a strict constraint, considering a single point mass model with a constant height:

$$
\boldsymbol{c}^{x, y}-\frac{c^{z}}{g^{z}} \ddot{\boldsymbol{c}}^{x, y} \in \operatorname{conv}\left\{\boldsymbol{p}_{i}^{x, y}\right\}
$$

and simply minimize the integral

$$
\min \int\left\|\dddot{\boldsymbol{c}}^{x, y}\right\|^{2} d t
$$


over a sufficiently long but finite length of time to ensure viability. This is the walking motion generation scheme implemented in the Aldebaran Nao robot [104].

A variant tested successfully on the DLR biped robot [105] considers minimizing the deviation from a reference trajectory for the $\mathrm{XCoM}$ together with the derivative of the CoP:

$$
\min \int\left\|\boldsymbol{\xi}^{x, y}-\boldsymbol{\xi}_{r e f}^{x, y}\right\|^{2}+\beta\left\|\dot{\boldsymbol{z}}^{x, y}\right\|^{2} d t
$$

An additional terminal constraint was considered but not tested.

A last proposition, tested only in simple simulations, not with a real robot [106], considers a single point mass which is assigned to minimize the deviation of the leg length $l$ from a given reference, and the deviation of the $\mathrm{CoP}$ from the middle of the contact points over the next two steps:

$$
\min \int\left(l-l_{\text {ref }}\right)^{2}+\beta\left\|\boldsymbol{c}^{x, y}-\frac{c^{z} \ddot{\boldsymbol{c}}^{x, y}}{\ddot{c}^{z}+g^{z}}-\overline{\boldsymbol{p}_{i}^{x, y}}\right\|^{2} d t .
$$

What makes this proposition particularly stimulating is that this is not an integral of the form (48.32), that can ensure viability, and no terminal constraint is there to impose capturability, so there is no direct relation with the viability/capturability analysis developed in Section 48.3.3, on which all the previous schemes were based. What this minimization only imposes is the ability to realize two more steps in the future, with legs approximately at a nominal length: the situation in the end of these two steps is not controlled (no terminal constraint), so the robot might very well be falling afterwards. However, in the presented simulations, it appears that maintaining this ability to make two more steps in the future is a sufficient condition for generating stable walking and running motions. The conclusion is that terminal constraints or integrals of the form (48.32) are in fact not mandatory and could be relaxed altogether.

In all of these approaches, the footsteps were predefined and kept fixed, which is obviously a strong limitation on the robot's capacity to adapt to a changing environment or to strong perturbations.

Adaptive footsteps. In fact, adapting foot placement is straightforward, and has already been validated on a Kawada HRP-2 robot [107]: the only required change is to consider foot placement as a decision variable, used in addition to the horizontal motion of the CoM, in order to both satisfy the ODI (48.47) and minimize the integral

$$
\min \int\left\|\dot{\boldsymbol{c}}^{x, y}-\dot{\boldsymbol{c}}_{r e f}^{x, y}\right\|^{2} d t
$$

over a sufficiently long length of time to ensure viability (since this is clearly an integral of the form (48.32)), and have the CoM follow on top of that the reference velocity, $\dot{\boldsymbol{c}}_{r e f}^{x, y}$. Now, since the footstep placement is decided online, geometric feasibility needs to be checked online as well. A simple but effective option is to consider a polygonal approximation of the reachable volume of the CoM with respect to each foot on the ground [108]. 
Yet, the timing of the steps was still predefined, what has a huge influence on the reactivity of legged robots [60]. In order to adapt it as well, it has been proposed in [109] to minimize the combined integral

$$
\min \int\left\|\dot{\boldsymbol{c}}^{x, y}-\dot{\boldsymbol{c}}_{r e f}^{x, y}\right\|^{2}+\left\|\ddot{\boldsymbol{f}}^{x, y}\right\|^{2} d t
$$

with $\ddot{f}^{x, y}$ the horizontal acceleration of the feet. But this approach has been tested only in simple simulations so far. Moreover, the underlying optimization problem becomes nonlinear, what is significantly more involved to solve.

Another approach, quite unique, starts with a singular LQR design [110], considering the classical single point mass at a constant height, assigned to minimize the deviation of the CoP from a combination of a reference CoP and the XCoM:

$$
\min \int\left\|\boldsymbol{c}^{x, y}-\frac{c^{z}}{g^{z}} \ddot{\boldsymbol{c}}^{x, y}+\alpha \boldsymbol{z}_{\text {ref }}^{x, y}-(1+\alpha) \boldsymbol{\xi}^{x, y}\right\|^{2} d t
$$

for some $\alpha>0$. An interesting feature of this singular LQR design is that it can be solved analytically. And an interesting property of this singular objective function is that it can be reduced to zero, in which case we have

$$
\boldsymbol{c}^{x, y}-\frac{c^{z}}{g^{z}} \ddot{\boldsymbol{c}}^{x, y}=(1+\alpha) \boldsymbol{\xi}^{x, y}-\alpha \boldsymbol{z}_{r e f}^{x, y},
$$

what can be combined with the dynamics (48.35) of the XCoM to obtain a stable first order dynamics

$$
\dot{\boldsymbol{\xi}}^{x, y}=\alpha \omega\left(\boldsymbol{z}_{r e f}^{x, y}-\boldsymbol{\xi}^{x, y}\right)
$$

according to which the $\mathrm{XCoM}, \boldsymbol{\xi}$, is going to converge to $\boldsymbol{z}_{\text {ref }}^{x, y}$, and the $\mathrm{CoP}, \boldsymbol{z}$, as well according to (48.54). But in a very unusual twist, this LQR design is not used as is, and is inverted analytically to find under which condition does a piecewise constant trajectory of the $\mathrm{CoP}$ generate a non-diverging motion of the CoM. Unsurprisingly, this non-diverging condition ends up being a terminal constraint on the Capture Point/XCoM/diverging part of the dynamics, with trajectories corresponding in the end exactly to those found in [59]. But here, this terminal constraint is used in the end to decide online the footstep placement that will ensure viability, what is eventually validated experimentally with very strong perturbations.

All variants of a common design. The viability analysis of Section 48.3.3 attested the crucial importance of anticipation to avoid falling. We observe now that the walking and running motion generation schemes that power most of the greatest humanoid robots of today and yesterday, the Honda Asimo, the Toyota Partner, the Sony Qrio, the Aldebaran Nao, the Kawada HRP-2, the Tokyo University H7, the Munich University Johnny, the DLR biped, the long series of Waseda University humanoids and many others, are all structured around this 
necessity to anticipate, at least a couple steps ahead of time. Viability is secured then in different ways, always related to MPC theory, through capturability or through minimizing an integral of the form (48.32). In all cases, adopting the artificial synergy synthesis approach, focusing on the motion of the CoM of the robot with respect to contact points, considering that the rest of the motion can be handled more or less independently, is the key to enable efficient computations. All these motion generation schemes appear then to share the same general design: Model Predictive Control of the CoM of the robot with respect to contact points. The rest is details.

The compelling consequence of identifying this common design, is revealing the possibility to crossbreed all these approaches, retaining the best features of each: more precise multiple mass models, for generating both walking and running motions, with adaptive footstep placement and adaptive timing, sensor feedback, all in order to obtain in the end the ultimate robust and versatile online motion generation scheme. These are the next obvious steps.

Missing in this gallery of great legged robots, the Boston Dynamics biped and quadruped robots. They are without a doubt the legged robots exhibiting the most impressive dynamic motions today, robust and versatile. Exact details about their control algorithms are scarce, but various clues suggest that they might share the same design.

\subsubsection{Motion in constrained environments}

We have seen so far how to generate dynamic walking and running motions by considering the dynamic feasibility constraint (48.13) independently from any other concern. But on truly rough terrain or in cluttered spaces, kinematic constraints on the motion of the robot cannot be disregarded and can further complicate the problem. More precisely, kinematic constraints generally make the reachable space of the robot non-convex, so that deciding actions and motions with only a local view on the situation can quickly get the robot stuck in local loops or dead-ends. One way to decide actions and motions with a global view on the situation is with planning techniques, as described in Chapter 7 on Motion Planning and Chapter 47 on Motion Planning and Obstacle Avoidance. In the case of legged robots, three classes of problems of increasing complexity have been considered: dynamic manipulation without locomotion, locomotion on a flat ground with obstacles, and locomotion on rough terrain with complex contact transitions.

Dynamic manipulation without locomotion. In the case of manipulation tasks without locomotion, the only difference of legged robots with respect to more traditional manipulator robots is the dynamic feasibility constraint (48.13). Therefore, a mere application of the standard planning techniques described in Chapters 7 and 47 including this feasibility constraint has proved to be fully sufficient. But these techniques are strongly biased towards quasi-static motions. As a result, a sequence of statically stable postures is computed first, and the motion is smoothed and accelerated afterwards, finally 
taking into account dynamic feasibility [111]. Much more involved are the problems involving locomotion, which attracted therefore much more attention.

Legged locomotion on a flat ground with obstacles. If we consider that the problem of generating dynamic legged locomotion is properly solved thanks to the different methods described earlier in this Section, we can try to abstract its details and plan the navigation of the upper body of a legged robot between obstacles as for any other mobile robot, with the same planning techniques, regardless of the exact locomotion method. It has been shown indeed that legged robots present a form of small-space controllability, which means that their upper-body can follow any given path with any given accuracy [112], although this can require impractically quick steps. A different option to follow exactly any given path with the upper-body is to cross legs, as if walking on a balance beam, and some robots have been specifically designed to be able to realize such feats [113], but this can require impractically slow steps. Ruling out impractically slow or impractically quick steps, the legged locomotion may finally differ from the planned path and fail to avoid obstacles. In this case, iterative replanning techniques have been proposed [114].

In this approach, however, it is assumed that finally finding proper footholds to follow the path planned for the upper body is not a problem. Yet, the appeal of legged robots is to provide improved mobility on difficult terrain, situations where finding footholds is actually a problem. A solution then is to check the availability of proper footholds when planning the upper body motion [115], an approach recently refined to show how to obtain in the general case an exact equivalence between planning a continuous path and planning a sequence of footholds on a flat ground $[116,117]$.

A different approach is to plan directly the footholds with respect to obstacles on the ground, and generate afterwards the corresponding dynamic legged locomotion [118]. This has been applied successfully to long distance navigation with a tiered strategy [119], with computations fast enough to run online in changing environments [120], taking into account deterministically moving obstacles [121], on mildly rough terrain [122, 123, 124], using on-board sensing [125]. Obstacles above the ground can be considered then with a hybrid bounding box or more refined swept volumes [126, 127], or one can approximate the free-space with convex segmentation [128].

Legged locomotion with complex contact transitions. On truly rough terrain or in cluttered environments, legged locomotion may require much more complex contact situations and contact transitions than in standard walking or running, involving not only contacts between the feet and the ground, but all potential contact surfaces on the robot and on the environment. The variability of these situations is beyond comparison with the previous case on flat ground, and chances of pre-computations or simplifications are strongly lacking in respect. Notably, the kinematic and dynamic constraints cannot be tackled independently, and having to consider them simultaneously results in an overwhelming 
computational problem. As a result, a major effort has been to find a proper ordering of the different sub-problems in order to obtain a tractable problem in the end, finally opting for planning contacts before motion [129, 130, 131]. Complex real-life use-cases have been solved this way [132, 133, 134], but the computational requirements still limit our ability to achieve the versatility of locomotion possible on flat ground. Online adaptation to changing environments has yet to be convincingly demonstrated and remains an active area of research.

As noted before, the underlying planning techniques manifest a strong bias towards quasi-static motions: some post-processing is necessary to obtain a truly dynamic motion in the end. The same two options as in Section 48.4.1 have been proposed, standard trajectory optimization [135], or following the artificial synergy synthesis approach and computing first the motion of the CoM of the robot with respect to the different contact points, independently from the rest of the motion of the robot, which is computed only afterwards [21]. But the strong interdependency between kinematic and dynamic constraints defeats the core assumption of the artificial synergy synthesis approach in this case: dynamic feasibility can't always be handled independently from the rest of the motion of the robot, so there is a significant risk that this method fails finding in the end a feasible motion even if one exists.

Further discussion of all these problems in the specific case of humanoid robots can be found in Section 5 of Chapter 67 on Whole-Body Activities of Humanoid Robots, or in [136]. There is also a rich literature on gait selection for many-legged robots, starting as early as [137] but this is still an active area of research [138].

\subsubsection{Motion generation with limited computing resources}

The walking and running motion generation schemes presented so far in this Chapter all require significant computation, even when they consider only simplified dynamical models. Early legged robots, which had very limited computing resources, had to rely on much less demanding approaches. One option is to combine simple explicit rules, each one focusing on a different part of the overall motion. Another option is to try to mimic biological hypotheses on how animals generate and control their motion, what ended up in fact with somehow similar propositions.

Combining simple rules. Thanks to simplifying symmetries in biped or quadruped running gaits such as trot, pace and bound, the whole family of robots hopping in the MIT LegLab throughout the 1980s on one, two or four legs, in $2 \mathrm{D}$ or $3 \mathrm{D}$, could rely on the same simple control design [5]. The general idea is to apply simple control laws independently to the different parts of the overall locomotion task. The vertical oscillations were controlled by open-loop vertical thrusts, simply stabilized by mechanical energy losses. Since the angular momentum is conserved during flight phases, the body attitude was controlled only during stance phases, with standard PD control. The key to maintaining long-term balance, and controlling the global motion was foot placement. An 
elementary analysis of the motion of the CoM during the stance phase revealed the role of the so-called neutral foot position, which would make the stance phase perfectly symmetric. It is then just a matter of linear corrections of the foot position with respect to this neutral position to make the stance phase asymmetric in order to control the robot's speed and balance. The direct combination of these utterly simple control laws resulted in impressively robust and versatile locomotion.

The first biped robot that walked dynamically relied on an equally simple approach [4]. This robot emulated walking on stilts, with contact surfaces between the feet and the ground reduced to single points, so $\boldsymbol{z}=\boldsymbol{p}$. With a single, fixed contact point during single support phases (the double support phases were supposed negligible), the ODI (48.29) can be solved analytically: for $t \geq t_{0}$,

$$
\left[\begin{array}{c}
\boldsymbol{c}^{x, y}(t)-\boldsymbol{p}^{x, y}\left(t_{0}\right) \\
\dot{\boldsymbol{c}}^{x, y}(t)
\end{array}\right]=\boldsymbol{A}(t)\left[\begin{array}{c}
\boldsymbol{c}^{x, y}\left(t_{0}\right)-\boldsymbol{p}^{x, y}\left(t_{0}\right) \\
\dot{\boldsymbol{c}}^{x, y}\left(t_{0}\right)
\end{array}\right]
$$

with

$$
\boldsymbol{A}(t)=\left[\begin{array}{cc}
\cosh \omega\left(t-t_{0}\right) & \omega^{-1} \sinh \omega\left(t-t_{0}\right) \\
\omega \sinh \omega\left(t-t_{0}\right) & \cosh \omega\left(t-t_{0}\right)
\end{array}\right], \omega=\sqrt{\frac{g^{z}}{c^{z}}}
$$

If the next single support phase starts at time $t_{1}$ with a foot position $\boldsymbol{p}^{x, y}\left(t_{1}\right)$, we end up having

$$
\left[\begin{array}{c}
\boldsymbol{c}^{x, y}\left(t_{1}\right)-\boldsymbol{p}^{x, y}\left(t_{1}\right) \\
\dot{\boldsymbol{c}}^{x, y}\left(t_{1}\right)
\end{array}\right]=\boldsymbol{A}\left(t_{1}\right)\left[\begin{array}{c}
\boldsymbol{c}^{x, y}\left(t_{0}\right)-\boldsymbol{p}^{x, y}\left(t_{0}\right) \\
\dot{\boldsymbol{c}}^{x, y}\left(t_{0}\right)
\end{array}\right]+\left[\begin{array}{c}
\boldsymbol{p}^{x, y}\left(t_{0}\right)-\boldsymbol{p}^{x, y}\left(t_{1}\right) \\
0
\end{array}\right]
$$

which describes the motion of the CoM $c^{x, y}$ with respect to the contact points $\boldsymbol{p}^{x, y}$, from one step, starting at time $t_{k}$, to the next, starting at time $t_{k+1}$. This discrete time dynamical system can be controlled then with the foot placement $\boldsymbol{p}^{x, y}\left(t_{k}\right)$. Since it is linear, standard pole placement can be applied, yielding a standard PD control of foot placement. This simple approach has been successfully applied on more complex biped robots [139, 140].

The drawback of combining independent control laws is to lack coordination yielding potentially harsh, unrefined motion, but this approach demonstrates that stable locomotion is possible even with very limited control resources. Of primary importance here are foot placement and upper body attitude; the rest of the motion appears to be secondary, at least with respect to balance control. Pushing this observation further, it has been proposed, and validated in simulation, that a large variety of walking patterns could be used, and stabilized only with upper body attitude and foot placement control, both with simple PD control laws [141]. It is not surprising then that hand-designed motion patterns can be used successfully on robots as complex as the KAIST Hubo humanoid robot [142], although this may require delicate fine tuning.

Reducing even further the control requirements, the mechanics of the robots could be tuned so that passive motions would automatically land the feet on the appropriate locations with respect to stability, ending up with perfectly passive stable dynamic locomotion. This idea will be discussed more thoroughly in Section 48.6. 
Biomimetic motion generation. Current theories on locomotion control in animals include Central Pattern Generators (CPGs) and cascades of reflex motions, which interact and combine to generate the final motion. CPGs are tunable oscillators, generating synchronized quasi-cyclic motion patterns in response to simple control signals such as locomotion speed or turning angle [143]. In order to introduce such CPGs in legged robots, standard oscillators such as the Van der Pol equation or the Hopf oscillator have been proposed [144, 145], or more biologically inspired neural oscillators [146] (ironically, Central Pattern Generators often happen to be decentralized, composed of a collection of synchronized oscillators [147, 145, 143]).

But CPGs are intrinsically open-loop motion generators, so to stabilize and adapt the motion of the robot to their environment, limited feedback loops have been introduced, similar to the simple control laws discussed earlier in this Section, focusing on upper body attitude and foot placement [148, 147, 145]. Continuing with the biological analogy, these simple feedback loops have been called reflexes. Following ideas discussed more thoroughly in Chapter 13 on Behavior-Based Systems, the subsumption architecture has been proposed to build much more complex networks of reactive behaviors or reflexes [149], and has allowed a hexapod robot to walk successfully on mildly rough terrain without any careful planning of the motion [150].

Fundamentally the same decoupling approach. Being inspired by simple mechanical analyses or by animals, these approaches based on combinations of simple rules demonstrate that stable walking can be realized without much computation, at least in simple situations. More complex situations may require more refined motions and therefore more refined motion generation schemes, such as the ones discussed earlier in this Chapter. But it is striking to observe again the same focus here on the motion of the CoM of the robot with respect to the contact points, when controlling either the upper body attitude or the foot placement (or both), regardless of the rest of the motion, which can be generated by CPGs, Inverse Kinematics or any other approach, simple or complex. This is fundamentally the same decoupling as proposed earlier with the artificial synergy synthesis approach.

\subsection{Motion and Force Control}

Properly executing the walking and running motions computed in the previous Section requires precise control of the motion of the robot and of the contact forces with the environment, applying techniques from Chapters 8 and 9 on Motion and Force Control. But since legged robots are often complex mechanical systems with many degrees of freedom and some form of redundancy, techniques from Chapter 10 on Redundant Manipulators also need to be applied frequently. And in case of contact with multiple surfaces, problems of contact force distribution appear, requiring techniques similar to those found in Chapter 38 on Grasping. And in the opposite case, during flight phases, when the robot is not 
at all in contact with its environment, its dynamics appear to share important properties with free-floating space robots, requiring control techniques similar to those found in Chapter 55 on Space Robotics. All these techniques (and many more) are necessary to make legged robots walk and run efficiently. The purpose of this Section is to see how they connect and are implemented in the case of legged robots.

Whole-body motion. Humanoid and other legged robots are often complex mechanical systems with a large number of degrees of freedom in order to accomplish the various kinematic and dynamic tasks necessary for simultaneously achieving locomotion, perception and manipulation of the environment, interaction with humans, etc. Making use of all available degrees of freedom, and not only those in the legs, to achieve multiple simultaneous objectives, and not only locomotion, is usually called whole-body motion control (see for example Section 5 of Chapter 67 ). We have seen previously that locomotion mostly involves the motion of the $\mathrm{CoM} \boldsymbol{c}$ with respect to points $\boldsymbol{p}_{i}$ of contact with the environment. Other objectives involve end-effector motion for manipulation, gaze control for perception, motion of specific parts of the robot for obstacle avoidance, etc. In all of these cases, what needs to be controlled is Cartesian motion of different parts of the robot, rather than joint motion. Several control schemes have been proposed to do this, standard inverse kinematics followed by joint space control [139], Virtual Model Control [151], the Task Function approach [152, 153], Operational Space Control [154].

Interestingly, the last two options allow specifying different priorities to the different objectives assigned to the robot, so in case these objectives are not feasible jointly, the robot tries first to achieve the objectives with higher priority. In the case of legged robots, maintaining balance and avoiding falls appear to be of primary importance for the safety of both the robot and its environment, so being able to consider this objective with a higher priority can be crucial in this regard. These control schemes allow moreover some form of decoupling between the different objectives, further contributing to the artificial synergy synthesis approach introduced earlier in the previous Section. We are going therefore to continue focusing here exclusively on the locomotion objective, since the other ones can be considered more or less independently and are not specific to legged robots.

Flight phases. We have seen earlier in this Chapter that during flight phases, when a legged robot is not in contact with its environment, the motion of the CoM invariably follows a standard falling motion: there is no possibility to make it move in any other way. But we have seen that even though the angular momentum, $\boldsymbol{L}$, is constant during flight phases, the joint configuration, $\hat{\boldsymbol{q}}$, and the global orientation, $\boldsymbol{\theta}_{0}$, of the robot can still be driven concurrently together to any desired value thanks to the non-holonomy of the angular momentum (48.5). However, a famous theorem due to R.W. Brockett [155] implies that continuous time-invariant feedback control laws such as those proposed in $[156,157]$ are 
not able to do so. The solution is to use discontinuous or time-varying control laws [158], an approach well established in space robotics $[159,160]$.

In the case of running motions, it has been proposed to specifically adapt leg trajectories during flight phases in order to control both the joint configuration and the global orientation at the end of the flight phases [161]. It has been measured indeed that a simple difference in the height of a single step can result in 1 or 2 degrees of difference in the global orientation of the robot [8]. Albeit small, this effect is far from negligible. More thorough discussions of nonholonomic constraints and the impact of Brockett's theorem can be found in Chapter 49 on the Modeling and Control of Wheeled Mobile Robots or in [158].

Angular momentum. Either considering the ODI (48.13) when walking on a flat ground, or the Newton and Euler equations (48.3) and (48.4) in the general case, the dynamic feasibility of legged motions appears to be directly and entirely related to the motion of the CoM, $\boldsymbol{c}$, and the angular momentum, $\boldsymbol{L}$. It has been naturally proposed therefore to control specifically those two quantities concurrently $[162,163,164]$. Of course, since CoM motion and angular momentum are directly related to contact forces, $\boldsymbol{f}_{i}$, (or equivalently to the $\mathrm{CoP}, \boldsymbol{z})$, controlling the former is absolutely equivalent to controlling the latter. The question however is whether the angular momentum should be specifically controlled to some given value and what that value should be [163, 164].

Of course, since the angular momentum is directly related to contact forces, it should comply with the unilaterality and Coulomb friction limitations on contact forces (48.11) and (48.17). As a matter of fact, the regulation of angular momentum has been discussed only in quasi-static situations [162, 163, 164], where the reference value is clearly zero, which generally complies well with these limitations. And in the only case where walking was considered, the horizontal angular momentum, $\boldsymbol{L}^{x, y}$, that appears in the ODI (48.13) when walking on a flat horizontal ground, was explicitly not controlled [162]. It appears, in fact, that during walking motions, the question of which value the angular momentum should have is very intricate, because of its nonholonomy, but it is definitely not zero $[8,103]$.

Even in quasi-static situations, both joint configuration and global orientation can be controlled independently from the angular momentum; the connection between these different values is very subtle. As a result, controlling the angular momentum does not induce a control of either joint configuration or global orientation (due to its nonholonomy). The opposite is true however: controlling joint configuration and global orientation does induce a control of the angular momentum as a derived quantity. It has been argued furthermore that common whole body motion objectives such as manipulation or interaction with humans are more directly related to joint configuration and global orientation than to angular momentum [165]. As a matter of fact, disregarding the exact variations of the angular momentum has been the most frequent option so far $[139,151,152,153,154,165]$. In the end, it is not clear if specifically controlling the angular momentum can help controlling legged robots: this is 
still an open question.

CoM motion control. Putting the angular momentum aside, and considering only the simple linear dynamics (48.29) when walking on a flat ground, and more precisely its decomposition (48.34)-(48.35), we have seen that every state satisfying the condition (48.36), such that the capture point, $\boldsymbol{\xi}$, is in the support polygon, is capturable and can therefore be stabilized. If the capture point is strictly inside the support polygon, the CoM can even be stabilized to any reference position, $\boldsymbol{c}_{r e f}$, above the support polygon, with a simple linear feedback of the capture point:

$$
\boldsymbol{z}^{x, y}=\boldsymbol{c}_{r e f}^{x, y}+k\left(\boldsymbol{\xi}^{x, y}-\boldsymbol{c}_{r e f}^{x, y}\right),
$$

where the feedback gain, $k>1$, must be tuned in order to keep the CoP, $\boldsymbol{z}$, within the support polygon, ensuring dynamic feasibility of the motion. Indeed, using this feedback with the dynamics (48.35) yields a stable closed loop dynamics

$$
\dot{\boldsymbol{\xi}}^{x, y}=\omega(k-1)\left(\boldsymbol{c}_{r e f}^{x, y}-\boldsymbol{\xi}^{x, y}\right),
$$

according to which the capture point, $\boldsymbol{\xi}$, will converge to the reference position, $\boldsymbol{c}_{r e f}$, and the CoM, $\boldsymbol{c}$, will converge to it as a result of the stable dynamics (48.34). It has been shown that among all Proportional-Derivative (PD) feedback of the motion of the CoM

$$
\boldsymbol{z}^{x, y}=\boldsymbol{c}_{r e f}^{x, y}+k\left(\boldsymbol{c}^{x, y}+\lambda \dot{\boldsymbol{c}}^{x, y}-\boldsymbol{c}_{r e f}^{x, y}\right),
$$

the feedback (48.59) of the capture point, with a choice of $\lambda=\omega^{-1}$, is the only one that will successfully stabilize all capturable states [166]. It has been successfully applied to walking motions on the DLR biped robot [59].

Now, if there is a time-varying perturbation or error, $\varepsilon$, in our dynamics, such that

$$
\boldsymbol{c}^{x, y}-\frac{c^{z}}{g^{z}} \ddot{\boldsymbol{c}}^{x, y}=\boldsymbol{z}^{x, y}+\boldsymbol{\varepsilon},
$$

the PD feedback (48.61) can be shown to be robustly stable (input-to-state stable, to be precise) with respect to $\varepsilon$, but with $\lambda<\omega^{-1}$ and $k>\frac{1}{\lambda^{2} \omega^{2}}$ [167]. As discussed above, with this choice of $\lambda$, not all capturable states will be stabilized successfully, even if there is no perturbation. This is unfortunately a usual trade-off in robust control.

Force feedback. Motion control of legged robots without contact force sensing and feedback has been shown to be possible for simple quasi-static balancing tasks, using passivity based control [168]. However, force sensing and feedback is commonly used when realizing more dynamic motions such as walking or running. One of the first problems to overcome in this case is the compliance of both the robot and its environment, which easily generates destabilizing oscillations $[169,170,171,172]$. Contact force control schemes of various complexity have been proposed, but always with the goal of damping 
these undesired oscillations. The exact implementation of these force control schemes generally relies on a stiff lower-level joint motion control in order to enhance disturbance rejection, leading to admittance control implementations $[139,169,170,171,172,173]$. More details on such control schemes can be found in Chapter 9 on Force Control.

Now, these damping control schemes inevitably introduce some delay in the realization of the desired contact forces. One option is to show that the CoM motion control scheme described earlier, which entirely relies on contact forces, is robust to such delay [59]. Another option is to take this delay into account with a simple first-order dynamics of the CoP:

$$
\dot{z}=\omega_{\boldsymbol{z}}\left(\boldsymbol{z}_{d}-\boldsymbol{z}\right)
$$

and adapt the previous CoM motion control scheme accordingly [174, 175]:

$$
\boldsymbol{z}_{d}^{x, y}=\boldsymbol{c}_{r e f}^{x, y}+k\left(\boldsymbol{\xi}^{x, y}-\boldsymbol{c}_{r e f}^{x, y}\right)+k^{\prime}\left(\boldsymbol{z}^{x, y}-\boldsymbol{c}_{r e f}^{x, y}\right) .
$$

This introduces some force feedback directly in the CoM motion control through the measurement of the $\mathrm{CoP}, \boldsymbol{z}$, what can help improve the reactivity of the controller to perturbations.

Contact forces distribution In case of contact with multiple surfaces, computing contact forces, $\boldsymbol{f}_{i}$, that allow realizing the desired motion control scheme through the Newton and Euler equations (48.3) and (48.4), and yet comply with both the unilaterality condition (48.11) and the Coulomb friction model (48.17), can be slightly more involved. The standard approach is through optimization, and more precisely Quadratic Programming (QP) with linear constraints, using a faceted, linear approximation of the Coulomb friction model (48.17):

$$
\boldsymbol{A} \boldsymbol{f}_{i} \leq \boldsymbol{b}
$$

It has been proposed then to minimize when possible the norm of the joint torques $\boldsymbol{u}$ in order to reduce energy consumption [152],

$$
\min _{\boldsymbol{f}_{i}}\|\boldsymbol{u}\|^{2}
$$

or to balance the contact forces between the different contact points, either by minimizing the sum of their norms [165],

$$
\min _{\boldsymbol{f}_{i}} \sum_{i}\left\|\boldsymbol{f}_{i}\right\|^{2},
$$

or the norm of their difference [176],

$$
\min _{\boldsymbol{f}_{i}}\left\|\boldsymbol{f}_{\text {left }}-\boldsymbol{f}_{\text {right }}\right\|^{2}
$$

or minimizing the tangential forces in order to reduce the risk of slipping [177],

$$
\min _{\boldsymbol{f}_{i}} \sum_{i}\left\|\boldsymbol{f}_{i}^{x, y}\right\|^{2} \text {. }
$$


More general QP formulations have been proposed [178, 179], including hierarchies of kinematic and dynamic tasks and constraints [153].

But contact forces obtained in these ways are not explicitly kept away from the edges of the friction cone and unilaterality condition, so that the slightest perturbation may quickly lead to sliding or tipping, what can lead to serious issues. The simplest way to steadily improve the robustness of the robot to such perturbations is to introduce a security margin $w$ :

$$
\boldsymbol{A} \boldsymbol{f}_{i} \leq \boldsymbol{b}-w \mathbf{1},
$$

where $\mathbf{1}$ is a vector of ones, but there is no obvious way to decide which value would be appropriate. One option then is to try to maximize it with a Linear Program (LP):

$$
\max _{w, \boldsymbol{f}_{i}} w,
$$

improving as much as possible the robustness of the robot to perturbations by selecting contact forces $\boldsymbol{f}_{i}$ the farthest possible from the edges of the (faceted) friction cone and unilaterality condition. Note that the maximal value $w^{*}$ obtained in this way is the residual radius discussed in [19].

\subsection{Towards more efficient walking}

Examples of remarkably efficient locomotion are ubiquitous in nature. From sprinting cheetahs to leaping sifakas, the variety and beauty of animal motion has inspired roboticists for decades. Yet some of the most impressive examples of humanoid walking, e.g., Honda's Asimo or Boston Dynamics' Atlas, have employed high-impedance joint control to achieve stable locomotion. As a result, the fluidity of their motions is far from that of their biological counterpart. Indeed, these robots expend an order of magnitude more energy than a typical human while walking[180].

In contrast, the passive dynamic walking machines of McGeer and others $[6,184]$ have been able to achieve surprisingly human-like gaits without any actuation, relying solely upon the their carefully designed body dynamics. These machines moved down sloped terrain, relying upon the conversion of potential to kinetic energy to balance the losses introduced by impacts and friction. Subsequent examples of minimally-actuated bipeds capable of walking under their own power on flat terrain made the first steps toward realizing efficient walking machines $[180,185]$.

The existence of these robots leads to several interesting questions. For example, how can we find limit cycles of underactuated walkers and reason about their stability? Simple models of walking systems have been used to try to answer these questions for over two decades. Such analyses typically rely upon linearization of the model dynamics to integrate the equations of motion or numerical simulation of the nonlinear dynamics and analysis via Poincaré maps (Section 48.3.2). For example, the compass gait model is one of the simplest walking models, consisting of three point masses - one at the hip and one on 


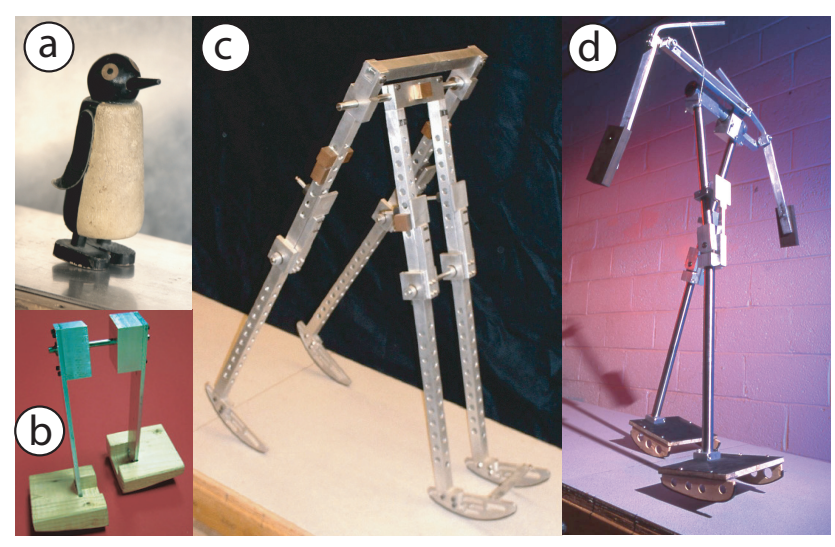

Figure 48.9: Passive-dynamic walking machines. (a) The Wilson Walkie [181]. (b) A slightly improved version [182]. Both (a) and (b) walk down a slight ramp with the comical, awkward, waddling gait of the penguin [181]. (c) Cornell copy [183] of McGeer's capstone design (7). This four-legged biped has two pairs of legs, an inner and outer pair, to prevent falling sideways. (d) The Cornell passive biped with arms (photo by Hank Morgan) [184]. This walker has knees and arms and is perhaps the most human-like passive-dynamic walker to date (8). (Photo reproduced with permissions from [180])

each leg - and the legs connected to the hip with a pin joint (Figure 48.10). Although even this very simple model cannot be analyzed analytically, a variety of stable gaits have been identified using numerical techniques [186].

Today there exists a community of researchers working to bridge the gap between stable and versatile machines like Asimo and efficient dynamic walkers. Topics of particular interest include navigating robustly over rough terrain, generating and stabilizing non-periodic gaits, and scaling up to more complex robot models. New computational tools for generating and stabilizing trajectories for underactuated dynamical systems have been an important result of this work. In this section we highlight the fundamental insights provided by work on passive dynamic walking machines and describe ways in which these ideas

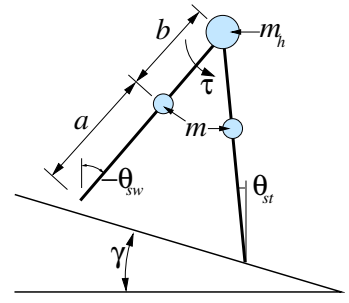

Figure 48.10: The compass gait 
have been extended to more general systems.

\subsubsection{Gait generation for dynamic walking}

The most direct approach to producing efficient walking gaits in underactuated robots is to design controllers that produce actuated trajectories that mimic passive ones. Fundamental to these methods is the idea of restoration of mechanical energy. Since mechanical energy is lost at each heel strike, passive dynamic walkers require gravitational force to restore energy to the system before the next step. By choosing control inputs that regulate mechanical energy, for example by simulating gravity, walking on level terrain can be achieved [187, 188]. However, such approaches are still largely limited to modest terrains and periodic motions.

Trajectory optimization methods offer the potential to move beyond limit cycle gaits and to more complicated systems without passive stability properties. However, the fact that walking systems must make and break contact with the world complicates the trajectory optimization problem. Simulating rigid-body contact forces continuously using spring and damper models often results in stiff differential equations that can lead to numerical and computational issues. An alternative approach is to capture discontinuities in velocity by modeling collisions as impulsive events using a hybrid system representation (Section 48.2.3). For a walking hybrid system, a set of autonomous guard functions are defined such that $\phi_{i}(\boldsymbol{q})=0$ implies that body $i$ is in contact and $\phi_{i}(\boldsymbol{q})>0$ implies the opposite. When a body comes into contact, the generalized velocity undergoes an instantaneous change, $\dot{\boldsymbol{q}}^{+}=f_{\Delta}\left(\boldsymbol{q}, \dot{\boldsymbol{q}}^{-}\right)$, where $\dot{\boldsymbol{q}}^{-}$and $\dot{\boldsymbol{q}}^{+}$are the velocities pre- and post-impact, respectively. The mode of the system is defined by the set of active contacts, $\mathcal{C}=\left\{i \mid \phi_{i}(\boldsymbol{q})=0\right\}$, which in turn defines the set of Jacobian matrices in (48.2).

The hybrid system representation creates several challenges for trajectory optimization methods. The discontinuities due to mode transitions are typically be accounted for explicitly using either direct or multiple-shooting methods[189]. Since each mode implies a different dynamics and mode changes are necessary for walking, this leads to the question of how to formulate the dynamics constraints. One solution is to predefine the sequence of mode transitions. For simple models with point feet, this approach can work quite well. However, for more complex models with many contacts, the number of modes grows exponentially, making mode sequence pre-specification impractical.

The complementarity-based methods for simulating rigid body systems in contact described in Section 48.2.3 suggest another approach. By discretizing the dynamics in time and considering only the integral of contact forces over a time interval, the forward dynamics of a walking system can be greatly simplified. Since many trajectory optimization methods already discretize by evaluating a finite set of points along a trajectory, incorporation of contact forces as optimization variables leads to trajectory optimization as a nonlinear optimization problem with complementarity constraints [37]. 
Many impressive examples of trajectory optimization for walking and running exist in the literature. For example, Mombaur [78] simultaneously optimized motion and physical parameters for simulated bipeds to achieve open-loop stable running and Remy [190] applied trajectory optimization to generate efficient running for a quadruped. There are many more examples and it remains an active area of research. The problem of stabilizing trajectories optimized offline remains a challenge, particularly when the execution deviates from the planned mode sequence. Computational requirements still prevent nonlinear trajectory optimization methods from being applied online as an MPC algorithm, but improvements in numerical solvers and computing hardware will likely continue to reduce this gap.

\subsubsection{Orbital trajectory stabilization and control}

Once an open-loop gait is generated, it must typically be stabilized in order to be executed on a robot. Classical techniques for trajectory stabilization from linear control can work, but a strong theme in stabilization for legged robots has been the idea that one should not enforce the precise timing of the trajectory. Section 48.3.2 discussed the notion of orbital stability and introduced Poincaré maps as a tool for stability analysis. Orbital stabilization, rather than traditional trajectory stabilization, sacrifices little and appears to be more compatible with underactuated robots. These ideas have also played an important role in developing stable dynamic walking and running controllers using virtual constraints [38]. Virtual constraints are holonomic constraints on monotonic functions of the robot's configuration variables. For example, for a forward periodic gait, it might be reasonable to assume that the angle of the stance leg with respect to the ground, $\theta_{s t}$, is monotonically increasing in time throughout the stance phase. The remaining configuration variables are written as functions of $\theta_{s t}$, effectively reparametrizing time, and constraints are imposed that, e.g., enforce symmetry in stance and swing leg angles $\left(\theta_{s w}=-\theta_{s t}\right)$ or coordinate arm and leg motion. This leads to outputs

$$
\boldsymbol{y}=\left[\begin{array}{c}
h_{1}(\boldsymbol{q}) \\
\ldots \\
h_{d}(\boldsymbol{q})
\end{array}\right],
$$

where each $h_{i}(\boldsymbol{q})$ encodes a virtual constraint. For example, $h(\boldsymbol{q})=\theta_{s w}+\theta_{s t}$ encodes the virtual constraint for swing-stance leg symmetry. By driving the output dynamics asymptotically to 0 using high-gain control, the system can be seen to evolve according to the virtual constraints, yielding a lower dimensional problem for control design and stability analysis. This approach has been used to produce remarkable dynamic walking and running examples in real robots [191].

Poincaré maps are defined with respect to a transverse surface at a single point on a periodic orbit, which limits their usefulness for designing stabilizing controllers. The transverse dynamics, on the other hand, is defined with respect to a continuous family of surfaces, $S(t)$, transverse to the orbit [45]. In the 


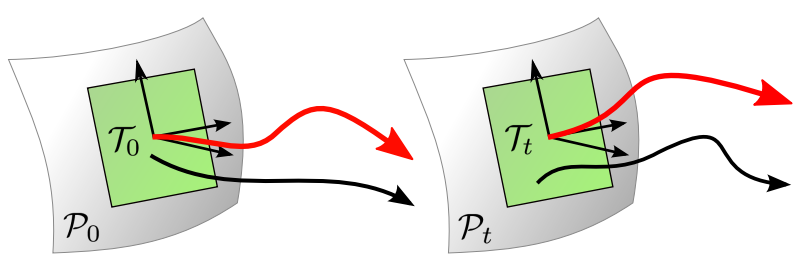

Figure 48.11: A simple illustration of transverse linearization along a nominal trajectory (red) for systems with impacts.

neighborhood of these coordinates, the dynamics can be written as

$$
\begin{aligned}
\dot{\tau} & =1+f_{1}\left(\tau, \boldsymbol{x}_{\perp}\right) \\
\dot{\boldsymbol{x}}_{\perp} & =\boldsymbol{A}(\tau) \boldsymbol{x}_{\perp}+f_{2}\left(\tau, \boldsymbol{x}_{\perp}\right),
\end{aligned}
$$

where $\tau \in\left[0, t_{f}\right]$ represents the orbit phase, $\boldsymbol{x}_{\perp}$ are the $2 N-1$ coordinates orthogonal to the flow of the system, and $f_{1}(\cdot), f_{2}(\cdot)$ are functions containing nonlinear terms. Note that unlike the discrete Poincaré map, these dynamics do not require the trajectory of interest to be a periodic orbit.

By extracting the linear part of the transversal dynamics and incorporating control inputs, we obtain the transverse linearization:

$$
\dot{\boldsymbol{z}}(t)=\boldsymbol{A}(t) \boldsymbol{z}(t)+\boldsymbol{B}(t) \boldsymbol{u}(t),
$$

for $t \in\left[0, t_{f}\right]$. Stabilization of this system, and therefore the underlying trajectory, can be approached using standard techniques from linear control theory. For walking systems with impulses, the dynamics can be extended to include a linearized impact map, $\boldsymbol{z}\left(t_{i}^{+}\right)=\boldsymbol{C}_{i} \boldsymbol{z}\left(t_{i}^{-}\right)$, where $t_{i}, i \in \mathcal{C}$ are times at which impacts occur. Figure 48.11 illustrates the idea of transversal surfaces about a trajectory for a system with impacts. Manchester et al. [50] showed that under some mild assumptions, local exponential orbital stability of planned motions on a hybrid nonlinear system can be achieved using a receding horizon control with the transverse linear dynamics.

Minor variations in impact timing can be handled in linearization-based approaches to stabilizing walking trajectories, but perturbations that lead to changes in mode ordering often cannot. To address this problem, we need algorithms for adjusting walking trajectories online. As described in the previous section, the hybrid nature of walking systems makes this challenging. One way to avoid this issue is to employ smooth approximations of the dynamics that permit the use of efficient local trajectory optimization techniques at the expense of violating complementarity condition (48.22) by, e.g., allowing interpenetration of rigid bodies. This approach has led to impressive examples of online trajectory optimization for simulated systems [192], but it is yet unknown how well these approximations will transfer to physical systems. 


\subsection{Different Contact Behaviors}

Contact forces have appeared throughout this Chapter to be central for the modeling and control of legged robots. As a result, a different contact behavior can result in a significantly different dynamical behavior of the whole robot, and this can be extremely useful in situations where standard walking or running is inefficient or downright impossible.

Wall climbing. Wall climbing legged robots rely on various devices such as suction cups, magnets, adhesive materials, miniature spine arrays, that can generate adhesive forces in order to stick to various vertical surfaces such as glass, steel, concrete, brick, stone $[193,194,195]$. In this case, the unilaterality condition (48.11), which was central throughout this Chapter, is no more relevant. As a result, the whole locomotion modeling and control is deeply transformed.

Tethered walking. On steep slopes, rappelling is an interesting option, tethering the robot to anchors in order to avoid tumbling down. This has been experienced successfully on construction sites [196], or in a volcano in the case of the impressive CMU Dante II octopod robot [197]. In this case, the locomotion problem is similar to the general case described throughout this Chapter, but the tether introduces an additional contact between the robot and the environment, on which the robot can pull but not push, so a unilateral contact exactly opposite to the standard unilateral contact between the feet and the ground, what can be treated however in exactly the same way.

Legs with wheels. Adapting wheeled vehicles to rough terrain has led in some cases to implant wheels on legs, combining the flexibility of articulated legs on rough terrain with the efficiency of wheels on flat terrain. Different options are possible, passive or active wheels, passive or active legs [198, 199, 200, 201, 202]. In this case, the unilaterality condition (48.11) is fundamental like in the rest of this Chapter, but the contact points can move freely on the contact surfaces along the direction of the wheels, what can significantly increase the array of possible motions [203]. Problems similar to those of standard legged robots include finding stable postures and allocating contact forces for maximizing stability and efficiency [200, 201, 202]. Interestingly, the case of passive wheels results in motions similar to skating [198].

Wheels with legs. Evolving the mechanical design of legs for greater simplicity and improved efficiency has led in some cases to directly implant the legs on wheels $[204,205]$. This results in very impressive outdoor performance, but not much related to standard legged robots in the end. 


\subsection{Conclusion}

Legged systems are at the heart of some of the most exciting work in modern robotics. They offer the opportunity to travel to places beyond the reach of wheeled systems and gain fundamental insights into the conditions under which stable and efficient locomotion is possible. At the same time, their complex dynamics pose significant challenges for our computational approaches to control and stability analysis. Indeed, the simple fact that every walking system must engage in intermittent contact with its environment has significant mathematical implications.

A large number of success stories for legged systems moving with remarkable precision and reliability have been witnessed over the past decade, and the development of exciting new tools for optimization-based planning, control, and analysis offers the promise to develop machines that achieve even higher levels of robustness and efficiency. 


\section{Bibliography}

[1] K. J. Waldron, R. B. McGhee: The Adaptive Suspension Vehicle, IEEE Control Systems Magazine 6, 7-12 (1986)

[2] K. Hirai, M. Hirose, Y. Haikawa, T. Takenaka: The Development of Honda Humanoid Robot, Proceedings of the IEEE International Conference on Robotics \& Automation 1998 1321-1326

[3] H. Lim, A. Takanishi: Biped walking robots created at Waseda University: WL and WABIAN family, Philosophical Transactions of the Royal Society A 365(1850), 49-64 (2007)

[4] H. Miura, I. Shimoyama: Dynamic Walk of a Biped, International Journal of Robotics Research 3(2), 60-74 (1984)

[5] M. Raibert: Legged Robots that Balance (MIT Press, Cambridge, 1986)

[6] T. McGeer: Passive Dynamic Walking (Simon Fraser University Technical Report, 1988)

[7] M. Raibert, K. Blankespoor, G. Nelson, R. Playter, the BigDog Team: BigDog, the Rough-Terrain Quadruped Robot, Proceedings of the IFAC World Congress 2008

[8] P.-B. Wieber: Holonomy and nonholonomy in the dynamics of articulated motion, Proceedings of the Ruperto Carola Symposium on Fast Motion in Biomechanics and Robotics 2005

[9] R. M. Murray, Z. Li, S. S. Sastry: A Mathematical Introduction to Robotic Manipulation (CRC Press, Inc., 1994)

[10] M. K. Vukobratović: Contribution to the Study of Anthropomorphic Systems, Kybernetika 8(5), 404-418 (1972)

[11] P. Sardain, G. Bessonnet: Forces Acting on a Biped Robot. Center of PressureZero Moment Point, IEEE Transactions on Systems, Man, and Cybernetics - Part A 34(5), 630-637 (september 2004) 
[12] K. Harada, S. Kajita, K. Kaneko, H. Hirukawa: ZMP Analysis for Arm/Leg Coordination, Proceedings of the IEEE/RSJ International Conference on Intelligent Robots \& Systems 2003 75-81

[13] K. Harada, H. Hirukawa, F. Kanehiro, K. Fujiwara, K. Kaneko, S. Kajita, M. Nakamura: Dynamical Balance of a Humanoid Robot Grasping an Environment, Proceedings of the IEEE/RSJ International Conference on Intelligent Robots \& Systems $20041167-1173$

[14] Y. Or, E. Rimon: Analytic Characterization of a Class of 3-Contact Frictional Equilibrium Postures in 3D Gravitational Environments, International Journal of Robotics Research (2010)

[15] P.-B. Wieber: On the stability of walking systems, Proceedings of the International Workshop on Humanoids and Human Friendly Robots 2002

[16] T. Saida, Y. Yokokoji, T. Yoshikawa: FSW (Feasible Solution of Wrench) for Multi-legged Robots, Proceedings of the IEEE International Conference on Robotics \& Automation 2003

[17] H. Hirukawa, S. Hattori, K. Harada, S. Kajita, K. Kaneko, F. Kanehiro, K. Fujiwara, M. Morisawa: A Universal Stability Criterion of the Foot Contact of Legged Robots - Adios ZMP, Proceedings of the IEEE International Conference on Robotics \& Automation 2006 1976-1983

[18] T. Bretl, S. Lall: Testing Static Equilibrium for Legged Robots, IEEE Transactions on Robotics 24(4), 794-807 (august 2008)

[19] S. Barthélemy, P. Bidaud: Stability Measure of Postural Dynamic Equilibrium based on Residual Radius, Proceedings of the International Symposium on Advances in Robot Kinematics 2008

[20] Z. Qiu, A. Escande, A. Micaelli, T. Robert: Human motions analysis and simulation based on a general criterion of stability, Proceedings of the International Symposium on Digital Human Modeling 2011

[21] Z. Qiu, A. Escande, A. Micaelli, T. Robert: A Hierarchical Framework for Realizing Dynamically-stable Motions of Humanoid Robot in Obstaclecluttered Environments, Proceedings of the IEEE-RAS International Conference on Humanoid Robots 2012

[22] E. Rimon, R. Mason, J. W. Burdick, Y. Or: A General Stance Stability Test Based on Stratified Morse Theory with Application to Quasi-Static Locomotion Planning, IEEE Transactions on Robotics 24(3), 626-641 (2008)

[23] Q. Huang, S. Sugano, K. Tanie: Stability Compensation of a Mobile Manipulator by Manipulator Motion: Feasibility and Planning, Proceedings of the IEEE/RSJ International Conference on Intelligent Robots \& Systems $19971285-1192$ 
[24] J. Kim, W. K. Chung, Y. Youm, B. H. Lee: Real-time ZMP Compensation Method using Null Motion for Mobile Manipulators, Proceedings of the IEEE International Conference on Robotics \& Automation 20021967 1972

[25] B. Brogliato: Nonsmooth Mechanics (Communications and Control Engineering Series, Springer-Verlag, London, 1999)

[26] F. Pfeiffer, C. Glocker: Multibody Dynamics with Unilateral Contacts (Wiley, New York, 1996)

[27] S. Chareyron, P.-B. Wieber: Stability and regulation of nonsmooth dynamical systems (INRIA Research Report RR-5408, 2004)

[28] C. Liu, Z. Zhao, B. Brogliato: Frictionless multiple impacts in multibody systems. I. Theoretical framework, Proceedings of the Royal Society A 464, 3193-3211 (2008)

[29] Y.-B. Jia, M. Mason, M. Erdmann: Multiple impacts: A state transition diagram approach, International Journal of Robotics Research (2012)

[30] J.-M. Bourgeot, C. Canudas de Wit, B. Brogliato: Impact shaping for double support walk: From the Rocking Block to the Biped Robot, Proceedings of the International Conference on Climbing and Walking Robots 2005

[31] B. Gamus, Y. Or: Analysis of dynamic bipedal robot locomotion with stick-slip transitions, Submitted to the IEEE International Conference on Robotics \& Automation 2013

[32] S. Kajita, K. Miura, M. Morisawa, K. Kaneko, F. Kanehiro, K. Yokoi: Evaluation of a Stabilizer for Biped Walk with Toe Support Phase, Proceedings of the IEEE-RAS International Conference on Humanoid Robots 2012

[33] V. Acary, B. Brogliato: Numerical Methods for Nonsmooth Dynamical Systems (Lecture Notes in Applied and Computational Mechanics Vol. $35,2008)$

[34] R. I. Leine, N. van de Wouw: Stability and Convergence of Mechanical Systems with Unilateral Constraints (Lecture Notes in Applied and Computational Mechanics Vol. 36, 2008)

[35] Y. Or, A.D. Ames: Stability and Completion of Zeno Equilibria in Lagrangian Hybrid Systems, IEEE Transactions on Automatic Control 56(6), $1322-1336$ (2011)

[36] D. E. Stewart, J. C. Trinkle: An Implicit time-stepping scheme for rigid body dynamics with inelastic collisions and coulomb friction, International Journal for Numerical Methods in Engineering 39(15), 2673-2691 (1996) 
[37] M. Posa, C. Cantu, R. Tedrake: A direct method for trajectory optimization of rigid bodies through contact, International Journal of Robotics Research 33(1), $69-81$ (2014)

[38] E. R. Westervelt, J. W. Grizzle, C. Chevallereau, J. H. Choi, B. Morris: Feedback Control of Dynamic Bipedal Robot Locomotion (CRC Press, Boca Raton, FL, 2007)

[39] M. Wisse: Essentials of dynamic walking; Analysis and design of twolegged robots $\mathrm{Ph} . \mathrm{D}$. Thesis 2004)

[40] R. Tedrake, I. R. Manchester, M. M. Tobenkin, J. W. Roberts: LQRTrees: Feedback Motion Planning via Sums of Squares Verification, International Journal of Robotics Research 29, 1038 - 1052 (2010)

[41] M. Posa, M. Tobenkin, R. Tedrake: Lyapunov Analysis of Rigid Body Systems with Impacts and Friction via Sums-of-Squares, Proceedings of the International Conference on Hybrid Systems: Computation and Control $201363-72$

[42] S. Cotton, I. Olaru, M. Bellman, T. van der Ven, J. Godowski, J. Pratt: FastRunner: A Fast, Efficient and Robust Bipedal Robot. Concept and Planar Simulation., Proceedings of the IEEE International Conference on Robotics \& Automation 2012

[43] M. Srinivasan, A. Ruina: Computer Optimization of a Minimal Biped Model Discovers Walking and Running, Nature 439, 72 - 75 (2006)

[44] K. Mombaur, H. G. Bock, J. P. Schloder, R. W. Longman: Open-loop stable solutions of periodic optimal control problems in robotics, Z. Angew. Math. Mech. (ZAMM) 85(7), $499-515$ (2005)

[45] J. Hauser, C. C. Chung: Converse Lyapunov functions for exponentially stable periodic orbits, Systems and Control Letters 23(1), 27 - 34 (1994)

[46] J. Guckenheimer, P. Holmes: Nonlinear Oscillations, Dynamical Systems, and Bifurcations of Vector Fields (Springer, 1983)

[47] I. R. Manchester, M. M. Tobenkin, M. Levashov, R. Tedrake: Regions of Attraction for Hybrid Limit Cycles of Walking Robots, Proceedings of the IFAC World Congress 2011

[48] E. R. Westervelt, G. Buche, J. W. Grizzle: Experimental Validation of a Framework for the Design of Controllers that Induce Stable Walking in Planar Bipeds, The International Journal of Robotics Research 23(6), $559-582(2004)$

[49] A. S. Shiriaev, L. B. Freidovich, I. R. Manchester: Can We Make a Robot Ballerina Perform a Pirouette? Orbital Stabilization of Periodic Motions of Underactuated Mechanical Systems, Annual Reviews in Control 32(2), $200-211(2008)$ 
[50] I. R. Manchester, U. Mettin, F. Iida, R. Tedrake: Stable dynamic walking over uneven terrain, International Journal of Robotics Research 30(3), $265-279$ (2011)

[51] J.-P. Aubin: Viability Theory (Birkhäuser, 1991)

[52] P.-B. Wieber: Constrained dynamics and parametrized control in biped walking, Proceedings of the International Symposium on Mathematical Theory of Networks and Systems 2000

[53] P.-B. Wieber: Viability and Predictive Control for Safe Locomotion, Proceedings of the IEEE/RSJ International Conference on Intelligent Robots \& Systems 2008

[54] K. Ogata: Modern Control Engineering, 3rd edn. (Prentice Hall Incorporated, 1996)

[55] J. Pratt, R. Tedrake: Velocity Based Stability Margins for Fast Bipedal Walking, Proceedings of the Ruperto Carola Symposium on Fast Motion in Biomechanics and Robotics 2005

[56] A. L. Hof, M. G. J. Gazendam, W. E. Sinke: The condition for dynamic stability, Journal of Biomechanics 38, 1-8 (2005)

[57] J. Pratt, J. Carff, S. Drakunov, A. Goswami: Capture Point: A Step toward Humanoid Push Recovery, Proceedings of the IEEE-RAS International Conference on Humanoid Robots 2006

[58] T. Takenaka, T. Matsumoto, T. Yoshiike: Real Time Motion Generation and Control for Biped Robot -1st Report: Walking Gait Pattern Generation-, Proceedings of the IEEE/RSJ International Conference on Intelligent Robots \& Systems 2009

[59] J. Englsberger, C. Ott, M. A. Roa, A. Albu-Schäffer, G. Hirzinger: Bipedal walking control based on Capture Point dynamics, Proceedings of the IEEE/RSJ International Conference on Intelligent Robots \& Systems 2011

[60] T. Koolen, T. de Boer, J. Rebula, A. Goswami, J. Pratt: Capturabilitybased analysis and control of legged locomotion, Part 1: Theory and application to three simple gait models, International Journal of Robotics Research 31(9), 1094-1113 (2012)

[61] A. Papachristodoulou, S. Prajna: Robust Stability Analysis of Nonlinear Hybrid Systems, IEEE Transactions on Automatic Control 54(5), 10351041 (2009)

[62] P. Hanggi, P. Talkner, M. Borkovec: Reaction-rate theory: fifty years after Kramers, Reviews of Modern Physics 62(2), 251-342 (1990) 
[63] K. Byl: Metastable Legged-Robot Locomotion Ph.D. Thesis 2008)

[64] J. Steinhardt, R. Tedrake: Finite-time Regional Verification of Stochastic Nonlinear Systems, International Journal of Robotics Research 31(7), $901-923(2012)$

[65] D. G. E. Hobbelen, M. Wisse: A Disturbance Rejection Measure for Limit Cycle Walkers: The Gait Sensitivity Norm, IEEE Transactions on Robotics 23(6), $1213-1224$ (2007)

[66] C. Ebenbauer: Polynomial Control Systems: Analysis and Design via Dissipation Inequalities and Sum of Squares (Universität Stuttgart PhD thesis, 2005)

[67] H. Dai, R. Tedrake: L2-Gain Optimization for Robust Bipedal Walking on Unknown Terrain, Proceedings of the IEEE International Conference on Robotics \& Automation 2013

[68] E. Garcia, J. Estremera, P. Gonzalez de Santos: A classification of stability margins for walking robots, Proceedings of the International Conference on Climbing and Walking Robots 2002

[69] A. Goswami: Postural stability of biped robots and the foot rotation indicator (FRI) point, International Journal of Robotics Research 18(6) (1999)

[70] C. K. Chow, D. H. Jacobson: Studies of human locomotion via optimal programming (Harvard University Technical Report No. 617, 1970)

[71] P. H. Channon, S. H. Hopkins, D. T. Phan: Derivation of optimal walking motions for a biped walking robot, Robotica 10(2), 165-172 (1992)

[72] G. Cabodevilla, N. Chaillet, G. Abba: Energy-Minimized Gait for a Biped Robot, Proceedings of Autonome Mobile Systeme 1995

[73] C. Chevallereau, A. Formal'sky, B. Perrin: Low energy cost reference trajectories for a biped robot, Proceedings of the IEEE International Conference on Robotics \& Automation 1998

[74] M. Rostami, G. Bessonnet: Impactless sagittal gait of a biped robot during the single support phase, Proceedings of the IEEE International Conference on Robotics \& Automation 1998

[75] L. Roussel, C. Canudas de Wit, A. Goswami: Generation of Energy Optimal Complete Gait Cycles for Biped Robots, Proceedings of the IEEE International Conference on Robotics \& Automation 1998

[76] J. Denk, G. Schmidt: Synthesis of a Walking Primitive Database for a Humanoid Robot using Optimal Control Techniques, Proceedings of the IEEE-RAS International Conference on Humanoid Robots 2001 
[77] T. Buschmann, S. Lohmeier, H. Ulbrich, F. Pfeiffer: Optimization Based Gait Pattern Generation for a Biped Robot, Proceedings of the IEEE-RAS International Conference on Humanoid Robots 2005

[78] K. Mombaur: Using optimization to create self-stable human-like running, Robotica 27(3), $321-330$ (2009)

[79] J. Denk, G. Schmidt: Synthesis of Walking Primitive Databases for Biped Robots in 3D-Environments, Proceedings of the IEEE International Conference on Robotics \& Automation 2003

[80] S. A. Setiawan, S. H. Hyon, J. Yamaguchi, A. Takanishi: Quasi Real-time Walking Control of a Bipedal Humanoid Robot Based on Walking Pattern Synthesis, Proceedings of the International Symposium on Experimental Robotics 1999

[81] P.-B. Wieber, C. Chevallereau: Online adaptation of reference trajectories for the control of walking systems, Robotics and Autonomous Systems $\mathbf{5 4}(7), 559-566(2006)$

[82] C. Liu, C. G. Atkeson: Standing balance control using a trajectory library, Proceedings of the IEEE/RSJ International Conference on Intelligent Robots \& Systems $20093031-3036$

[83] J. Yamaguchi, A. Takanishi, I. Kato: Development of Biped Walking Robot Compensating for Three-Axis Moment by Trunk Motion, Proceedings of the IEEE/RSJ International Conference on Intelligent Robots \& Systems 1993

[84] Q. Huang, K. Yokoi, S. Kajita, K. Kaneko, H. Arai, N. Koyachi, K. Tanie: Planning Walking Patterns for a Biped Robot, IEEE Transactions on Robotics and Automation 17(3), 280-289 (june 2001)

[85] P.-B. Wieber: Trajectory Free Linear Model Predictive Control for Stable Walking in the Presence of Strong Perturbations, Proceedings of the IEEE-RAS International Conference on Humanoid Robots 2006

[86] T. de Boer: Foot placement in robotic bipedal locomotion (Technische Universiteit Delft PhD thesis, 2012)

[87] R. J. Full, D. E. Koditschek: Templates and Anchors: Neuromechanical Hypotheses of Legged Locomotion on Land, Journal of Experimental Biology 202, 3325-3332 (1999)

[88] R. McN. Alexander: Mechanics of bipedal locomotion, Perspectives in Experimental Biology 1, 493-504 (1976)

[89] R. McN. Alexander: Simple Models of Human Movement, ASME Applied Mechanics Reviews 48(8), 461-470 (1995) 
[90] S. S. Keerthi, E. G. Gilbert: Optimal Infinite-Horizon Feedback Laws for a General Class of Constrained Discrete-Time Systems: Stability and Moving-Horizon Approximations, Journal of Optimization Theory and Applications 57(2), 265-293 (1988)

[91] D. Q. Mayne, J. B. Rawlings, C. V. Rao, P. O. M. Scokaert: Constrained model predictive control: stability and optimality, Automatica 26(6), $789-814(2000)$

[92] M. Alamir, N. Marchand: Numerical stabilisation of non-linear systems: Exact theory and approximate numerical implementation, European Journal of Control 5(1), 87-97 (1999)

[93] M. Alamir, G. Bornard: Stability of a truncated infinite constrained receding horizon scheme: The general discrete nonlinear case, Automatica 31(9), 1353-1356 (1995)

[94] A. Takanishi, M. Tochizawa, H. Karaki, I. Kato: Dynamic biped walking stabilized with optimal trunk and waist motion, Proceedings of the IEEE/RSJ International Conference on Intelligent Robots \& Systems 1989

[95] H. Lim, Y. Kaneshima, A. Takanishi: Online Walking Pattern Generation for Biped Humanoid Robot with Trunk, Proceedings of the IEEE International Conference on Robotics \& Automation 2002

[96] T. Buschmann, S. Lohmeier, M. Bachmayer, H. Ulbrich, F. Pfeiffer: A Collocation Method for Real-Time Walking Pattern Generation, Proceedings of the IEEE-RAS International Conference on Humanoid Robots 2007

[97] K. Nishiwaki, S. Kagami, Y. Kuniyoshi, M. Inaba, H. Inoue: Online Generation of Humanoid Walking Motion based on a Fast Generation Method of Motion Pattern that Follows Desired ZMP, Proceedings of the IEEE/RSJ International Conference on Intelligent Robots \& Systems 2002

[98] R. Tajima, D. Honda, K. Suga: Fast Running Experiments Involving a Humanoid Robot, Proceedings of the IEEE International Conference on Robotics \& Automation 2009

[99] K. Nagasaka, Y. Kuroki, S. Suzuki, Y. Itoh, J. Yamaguchi: Integrated Motion Control for Walking, Jumping and Running on a Small Bipedal Entertainment Robot, Proceedings of the IEEE International Conference on Robotics \& Automation 2004

[100] M. Morisawa, K. Harada, S. Kajita, K. Kaneko, F. Kanehiro, K. Fujiwara, S. Nakaoka, H. Hirukawa: A Biped Pattern Generation Allowing Immediate Modification of Foot Placement in Real-time, Proceedings of the IEEE-RAS International Conference on Humanoid Robots 2006 
[101] S. Kajita, F. Kanehiro, K. Kaneko, K. Fujiwara, K. Harada, K. Yokoi, H. Hirukawa: Biped Walking Pattern Generation by using Preview Control of Zero Moment Point, Proceedings of the IEEE International Conference on Robotics \& Automation 2003 1620-1626

[102] K. Nishiwaki, S. Kagami: Online Walking Control Systems for Humanoids with Short Cycle Pattern Generation, International Journal of Robotics Research 28(6), 729-742 (2009)

[103] J. Park, Y. Youm: General ZMP Preview Control for Bipedal Walking, Proceedings of the IEEE International Conference on Robotics \& Automation 2007

[104] D. Gouaillier, C. Collette, C. Kilner: Omni-directional Closed-loop Walk for NAO, Proceedings of the IEEE-RAS International Conference on $\mathrm{Hu}-$ manoid Robots 2010

[105] M. Krause, J. Englsberger, P.-B. Wieber, C. Ott: Stabilization of the Capture Point Dynamics for Bipedal Walking based on Model Predictive Control, Proceedings of the IFAC Symposium on Robot Control 2012

[106] M. van de Panne: From footprints to animation, Computer Graphics 16(4), 211-223 (1997)

[107] A. Herdt, H. Diedam, P.-B. Wieber, D. Dimitrov, K. Mombaur, M. Diehl: Online Walking Motion Generation with Automatic Foot Step Placement, Advanced Robotics 24(5-6), 719-737 (2010)

[108] A. Herdt, N. Perrin, P.-B. Wieber: LMPC based online generation of more efficient walking motions, Proceedings of the IEEE-RAS International Conference on Humanoid Robots 2012

[109] Z. Aftab, T. Robert, P.-B. Wieber: Ankle, hip and stepping strategies for humanoid balance recovery with a single Model Predictive Control scheme, Proceedings of the IEEE-RAS International Conference on $\mathrm{Hu}-$ manoid Robots 2012

[110] J. Urata, K. Nishiwaki, Y. Nakanishi, K. Okada, S. Kagami, M. Inaba: Online Decision of Foot Placement using Singular LQ Preview Regulation, Proceedings of the IEEE-RAS International Conference on Humanoid Robots 2011

[111] J. Kuffner, S. Kagami, K. Nishiwaki, M. Inaba, H. Inoue: DynamicallyStable Motion Planning for Humanoid Robots, Autonomous Robots 12, $105-118$ (2002)

[112] S. Dalibard, A. El Khoury, F. Lamiraux, M. Taix, J.-P. Laumond: SmallSpace Controllability of a Walking Humanoid Robot, Proceedings of the IEEE-RAS International Conference on Humanoid Robots 2011 
[113] K. Kaneko, F. Kanehiro, S. Kajita, H. Hirukawa, T. Kawasaki, M. Hirata, K. Akachi, T. Isozumi: Humanoid Robot HRP-2, Proceedings of the IEEE International Conference on Robotics \& Automation 2004 1083-1090

[114] E. Yoshida, C. Esteves, I. Belousov, J.-P. Laumond, T. Sakaguchi, K. Yokoi: Planning 3-D Collision-Free Dynamic Robotic Motion Through Iterative Reshaping, IEEE Transactions on Robotics 24(5), 1186-1198 (october 2008)

[115] J.-D. Boissonnat, O. Devillers, S. Lazard: Motion planning of legged robots, SIAM Journal on Computing 30(1), 218-246 (2000)

[116] N. Perrin, O. Stasse, F. Lamiraux, E. Yoshida: Weakly collision-free paths for continuous humanoid footstep planning, Proceedings of the IEEE/RSJ International Conference on Intelligent Robots \& Systems 2011

[117] N. Perrin: From Discrete to Continuous Motion Planning, Proceedings of the International Workshop on the Algorithmic Foundations of Robotics 2012

[118] J. Kuffner, S. Kagami, K. Nishiwaki, M. Inaba, H. Inoue: Online Footstep Planning for Humanoid Robots, Proceedings of the IEEE International Conference on Robotics \& Automation 2003

[119] J. Chestnutt, J. Kuffner: A Tiered Planning Strategy for Biped Navigation, Proceedings of the IEEE-RAS International Conference on $\mathrm{Hu}-$ manoid Robots 2004

[120] P. Michel, J. Chestnutt, J. Kuffner, T. Kanade: Vision-Guided Humanoid Footstep Planning for Dynamic Environments, Proceedings of the IEEERAS International Conference on Humanoid Robots 2005

[121] J. Chestnutt, P. Michel, J. Kuffner, T. Kanade: Locomotion among dynamic obstacles for the Honda Asimo, Proceedings of the IEEE/RSJ International Conference on Intelligent Robots \& Systems 2007

[122] J.-M. Bourgeot, N. Cislo, B. Espiau: Path-Planning and Tracking in a 3D Complex Environment for an Anthropomorphic Biped Robot, Proceedings of the IEEE/RSJ International Conference on Intelligent Robots \& Systems 2002

[123] J. Chestnutt, J. Kuffner, K. Nishiwaki, S. Kagami: Planning Biped Navigation Strategies in Complex Environments, Proceedings of the IEEERAS International Conference on Humanoid Robots 2003

[124] M. Zucker, J. A. Bagnell, C. Atkeson, J. Kuffner: An Optimization Approach to Rough Terrain Locomotion, Proceedings of the IEEE International Conference on Robotics \& Automation 2010 
[125] J. Chestnutt, Y. Takaoka, K. Suga, K. Nishiwaki, J. Kuffner, S. Kagami: Biped Navigation in Rough Environments using On-board Sensing, Proceedings of the IEEE/RSJ International Conference on Intelligent Robots \& Systems 2009

[126] N. Perrin, O. Stasse, F. Lamiraux, Y. Kim, D. Manocha: Real-time footstep planning for humanoid robots among 3D obstacles using a hybrid bounding box, Proceedings of the IEEE International Conference on Robotics \& Automation 2012

[127] N. Perrin, O. Stasse, L. Baudoin, F. Lamiraux, E. Yoshida: Fast humanoid robot collision-free footstep planning using swept volume approximations, IEEE Transactions on Robotics 28(2), $427-439$ (2012)

[128] R. L. H. Deits, R. Tedrake: Computing Large Convex Regions of ObstacleFree Space through Semidefinite Programming, Proceedings of the International Workshop on the Algorithmic Foundations of Robotics 2014

[129] T. Bretl, S. Lall, J.-C. Latombe, S. Rock: Multi-Step Motion Planning for Free-Climbing Robots, Proceedings of the International Workshop on the Algorithmic Foundations of Robotics 2004

[130] K. Hauser, T. Bretl, J.-C. Latombe: Non-Gaited Humanoid Locomotion Planning, Proceedings of the IEEE-RAS International Conference on $\mathrm{Hu}-$ manoid Robots 2005

[131] K. Hauser, T. Bretl, J.-C. Latombe, K. Harada, B. Wilcox: Motion Planning for Legged Robots on Varied Terrain, International Journal of Robotics Research (2008)

[132] A. Escande, A. Kheddar, S. Miossec: Planning support contact-points for humanoid robots and experiments on HRP-2, Proceedings of the IEEE/RSJ International Conference on Intelligent Robots \& Systems 2006

[133] A. Escande, A. Kheddar, S. Miossec, S. Garsault: Planning support contact-points for acyclic motions and experiments on HRP-2, Proceedings of the International Symposium on Experimental Robotics 2008

[134] K. Bouyarmane, J. Vaillant, F. Keith, A. Kheddar: Exploring Humanoid Robots Locomotion Capabilities in Virtual Disaster Response Scenarios, Proceedings of the IEEE-RAS International Conference on Humanoid Robots 2012

[135] S. Lengagne, J. Vaillant, E. Yoshida, A. Kheddar: Generation of Wholebody Optimal Dynamic Multi-Contact Motions, International Journal of Robotics Research (2008)

[136] K. Harada, E. Yoshida, K. Yokoi: Motion Planning for Humanoid Robots (Springer, 2010) 
[137] R. B. McGhee, A. A. Frank: On the stability properties of quadruped creeping gaits, Mathematical Biosciences 3, 331 - 351 (1968)

[138] G. C. Haynes, A. A. Rizzi: Gaits and Gait Transitions for Legged Robots, Proceedings of the IEEE International Conference on Robotics \& Automation $20061117-1122$

[139] Y. Fujimoto, A. Kawamura: Proposal of Biped Walking Control Based on Robust Hybrid Position/Force Control,, 2724-2730

[140] S. Kajita, F. Kanehiro, K. Kaneko, K. Fujiwara, K. Yokoi, H. Hirukawa: A Realtime Pattern Generator for Biped Walking, Proceedings of the IEEE International Conference on Robotics \& Automation 200231 -37

[141] K. Yin, K. Loken, M. van de Panne: SIMBICON: Simple Biped Locomotion Control, Proceedings of ACM SIGGRAPH 2007

[142] I.-W. Park, J.-Y. Kim, J.-H. Oh: Online Biped Walking Pattern Generation for Humanoid Robot KHR-3(KAIST Humanoid Robot 3: HUBO), Proceedings of the IEEE-RAS International Conference on Humanoid Robots 2006

[143] A. Ijspeert, A. Crespi, D. Ryczko, J.-M. Cabelguen: From swimming to walking with a salamander robot driven by a spinal cord model, Science 315(5817), 1416-1420 (2007)

[144] R. Katoh, M. Mori: Control method of biped locomotion giving asymptotic stability of trajectory, Automatica 20(4), 405-414 (july 1984)

[145] L. Righetti, A. Ijspeert: Programmable Central Pattern Generators: an application to biped locomotion control, Proceedings of the IEEE International Conference on Robotics \& Automation 2006

[146] K. Matsuoka: Sustained Oscillations Generated by Mutually Inhibiting Neurons with Adaptation, Biological Cybernetics 52, 367-376 (1985)

[147] G. Endo, J. Morimoto, J. Nakanishi, G. Cheng: An Empirical Exploration of a Neural Oscillator for Biped Locomotion Control, Proceedings of the IEEE International Conference on Robotics \& Automation 20043036 3042

[148] Y. Fukuoka, H. Kimura, A. Cohen: Adaptive Dynamic Walking of a Quadruped Robot on Irregular Terrain Based on Biological Concepts, International Journal of Robotics Research 22(3-4), 187-202 (2003)

[149] R. Brooks: Elephants don't play chess, Robotics and Autonomous Systems 6, 3-15 (1990)

[150] R. Brooks: A Robot that Walks; Emergent Behaviors from a Carefully Evolved Network, Proceedings of the IEEE International Conference on Robotics \& Automation $1989292-296$ 
[151] J. Pratt, C.-M. Chew, A. Torres, P. Dilworth, G. Pratt: Virtual Model Control: An Intuitive Approach for Bipedal Locomotion, International Journal of Robotics Research 20, 129-143 (2001)

[152] F. Génot, B. Espiau: On the Control of the Mass Center of Legged Robots under Unilateral Constraints, Proceedings of the International Conference on Climbing and Walking Robots 1998

[153] L. Saab, O. E. Ramos, F. Keith, N. Mansard, P. Souères, J.-Y. Fourquet: Dynamic Whole-Body Motion Generation under Rigid Contacts and other Unilateral Constraints, To appear in IEEE Transactions on Robotics

[154] L. Sentis, J. Park, O. Khatib: Compliant Control of Multicontact and Center-of-Mass Behaviors in Humanoid Robots, IEEE Transactions on Robotics 26(3), $483-501$ (june 2010)

[155] R.W. Brockett: Asymptotic stability and feedback stabilization, Differential Geometric Control Theory 1983

[156] S. Kajita, T. Nagasaki, K. Kaneko, K. Yokoi, K. Tanie: A Running Controller of Humanoid Biped HRP-2LR, Proceedings of the IEEE International Conference on Robotics \& Automation 2005 618-624

[157] L. Sentis, O. Khatib: Control of Free-Floating Humanoid Robots Through Task Prioritization, Proceedings of the IEEE International Conference on Robotics \& Automation $20051730-1735$

[158] A. De Luca, G. Oriolo: Modelling and Control of Nonholonomic Mechanical Systems, Kinematics and Dynamics of Multi-Body Systems, CISM Courses and Lectures (1995)

[159] Y. Nakamura, R. Mukherjee: Exploiting Nonholonomic Redundancy of Free-Flying Space Robots, IEEE Transactions on Robotics and Automation 9(4), 499-506 (august 1993)

[160] E. Papadopoulos: Nonholonomic Behaviour in Free-floating Space Manipulators and its Utilization, Nonholonomic Motion Planning, Y. Xu and T. Kanade (Eds.) (1993)

[161] C. Chevallereau, E.R. Westervelt, J.W. Grizzle: Asymptotically Stable Running for a Five-Link, Four-Actuator, Planar Bipedal Robot, International Journal of Robotics Research 24(6), 431-464 (2005)

[162] S. Kajita, F. Kanehiro, K. Kaneko, K. Fujiwara, K. Harada, K. Yokoi, H. Hirukawa: Resolved Momentum Control: Humanoid Motion Planning based on the Linear and Angular Momentum, Proceedings of the IEEE/RSJ International Conference on Intelligent Robots \& Systems 2003 1644-1650 
[163] M. Popovic, A. Hofmann, H. Herr: Zero spin angular momentum control: definition and applicability, Proceedings of the IEEE-RAS International Conference on Humanoid Robots 2004

[164] S.-H. Lee, A. Goswami: Ground reaction force control at each foot: A momentum-based humanoid balance controller for non-level and nonstationary ground, Proceedings of the IEEE/RSJ International Conference on Intelligent Robots \& Systems $20103157-3162$

[165] C. Ott, M. A. Roa, G. Hirzinger: Posture and Balance Control for Biped Robots based on Contact Force Optimization, Proceedings of the IEEERAS International Conference on Humanoid Robots 2011

[166] T. Sugihara: Standing Stabilizability and Stepping Maneuver in Planar Bipedalism based on the Best COM-ZMP Regulator, Proceedings of the IEEE International Conference on Robotics \& Automation 2009

[167] Y. Choi, D. Kim, B.-J. You: On the Walking Control for Humanoid Robot based on the Kinematic Resolution of CoM Jacobian with Embedded Motion, Proceedings of the IEEE International Conference on Robotics \& Automation $20062655-2660$

[168] S.-H. Hyon: Compliant Terrain Adaptation for Biped Humanoids Without Measuring Ground Surface and Contact Forces, IEEE Transactions on Robotics 25(1), 171-178 (february 2009)

[169] S. Kajita, K. Yokoi, M. Saigo, K. Tanie: Balancing a Humanoid Robot Using Backdrive Concerned Torque Control and Direct Angular Momentum Feedback, Proceedings of the IEEE International Conference on Robotics \& Automation $20013376-3382$

[170] S. Lohmeier, K. Löffler, M. Gienger, H. Ulbrich, F. Pfeiffer: Computer System and Control of Biped "Johnnie", Proceedings of the IEEE International Conference on Robotics \& Automation $20044222-4227$

[171] J.-H. Kim, J.-H. Oh: Walking Control of the Humanoid Platform KHR-1 based on Torque Feedback Control, Proceedings of the IEEE International Conference on Robotics \& Automation $2004623-628$

[172] M.-S. Kim, J.-H. Oh: Posture Control of a Humanoid Robot with a Compliant Ankle Joint, International Journal of Humanoid Robotics 7(1), 529 (2010)

[173] T. Buschmann, S. Lohmeier, H. Ulbrich: Biped Walking Control Based on Hybrid Position/Force Control, Proceedings of the IEEE/RSJ International Conference on Intelligent Robots \& Systems 2009 3019-3024

[174] S. Kajita, M. Morisawa, K. Miura, S. Nakaoka, K. Harada, K. Kaneko, F. Kanehiro, K. Yokoi: Biped Walking Stabilization Based on Linear Inverted Pendulum Tracking, Proceedings of the IEEE/RSJ International Conference on Intelligent Robots \& Systems $20104489-4496$ 
[175] M. Morisawa, S. Kajita, F. Kanehiro, K. Kaneko, K. Miura, K. Yokoi: Balance Control based on Capture Point Error Compensation for Biped Walking on Uneven Terrain, Proceedings of the IEEE-RAS International Conference on Humanoid Robots 2012

[176] Y. Fujimoto, A. Kawamura: Simulation of an Autonomous Biped Walking Robot Including Environmental Force Interaction, IEEE Robotics and Automation Magazine 5(2), 33-41 (1998)

[177] L. Righetti, J. Buchli, M. Mistry, M. Kalakrishnan, S. Schaal: Optimal distribution of contact forces with inverse dynamics control, International Journal of Robotics Research 32(3), 280-298 (2013)

[178] A. D. Ames: Human-Inspired Control of Bipedal Robotics via Control Lyapunov Functions and Quadratic Programs, Proceedings of the International Conference on Hybrid Systems: Computation and Control 2013

[179] S. Kuindersma, F. Permenter, R. Tedrake: An Efficiently Solvable Quadratic Program for Stabilizing Dynamic Locomotion, Proceedings of the IEEE International Conference on Robotics \& Automation 2014

[180] S. H. Collins, A. Ruina, R. Tedrake, M. Wisse: Efficient Bipedal Robots Based on Passive-Dynamic Walkers, Science 307, 1082 - 1085 (2005)

[181] J. E. Wilson: Walking Toy (United States Patent Office, 1936)

[182] R. Tedrake, T. W Zhang, M. F. Fong, H. S. Seung: Actuating a Simple 3D Passive Dynamic Walker, Proceedings of the IEEE International Conference on Robotics \& Automation 20044656 - 4661

[183] M. Garcia, A. Chatterjee, A. Ruina: Efficiency, speed, and scaling of twodimensional passive-dynamic walking, Dynamics and Stability of Sytems 15(2), $75-99$ (2000)

[184] S. H. Collins, M. Wisse, A. Ruina: A Three-Dimensional Passive-Dynamic Walking Robot with Two Legs and Knees, International Journal of Robotics Research 20(7), 607-615 (2001)

[185] R. Tedrake, T. W. Zhang, H. S. Seung: Stochastic Policy Gradient Reinforcement Learning on a Simple 3D Biped, Proceedings of the IEEE/RSJ International Conference on Intelligent Robots \& Systems $20042849-$ 2854

[186] A. Goswami, B. Thuilot, B. Espiau: Compass-Like Biped Robot Part I : Stability and Bifurcation of Passive Gaits (INRIA Research Report No. 2996, 1996)

[187] M. W. Spong, G. Bhatia: Further Results on Control of the Compass Gait Biped, Proceedings of the IEEE/RSJ International Conference on Intelligent Robots \& Systems $20031933-1938$ 
[188] F. Asano, Z.-W. Luo, M. Yamakita: Biped Gait Generation and Control Based on a Unified Property of Passive Dynamic Walking, IEEE Transactions on Robotics 21(4), 754-762 (2005)

[189] J. T. Betts: Survey of Numerical Methods for Trajectory Optimization, Journal of Guidance, Control, and Dynamics 21(2), 193-207 (1998)

[190] C. D. Remy: Optimal Exploitation of Natural Dynamics in Legged Locomotion Ph.D. Thesis 2011)

[191] K. Sreenath, H. W. Park, I. Poulakakis, J. W. Grizzle: A compliant hybrid zero dynamics controller for stable, efficient and fast bipedal walking on MABEL, International Journal of Robotics Research (2010)

[192] Y. Tassa, T. Erez, E. Todorov: Synthesis and stabilization of complex behaviors through online trajectory optimization, Proceedings of the IEEE/RSJ International Conference on Intelligent Robots \& Systems 2012 $4906-4913$

[193] S. Hirose, A. Nagakubo, R. Toyama: Machine that can walk and climb on floors, walls and ceilings, Proceedings of the International Conference on Advanced Robotics $1991753-758$

[194] T. Yano, S. Numao, Y. Kitamura: Development of a self-contained wall climbing robot with scanning type suction cups, Proceedings of the IEEE/RSJ International Conference on Intelligent Robots \& Systems 1998 $249-254$

[195] S. Kim, A. Asbeck, W. Provancher, M. R. Cutkosky: SpinybotII: Climbing hard walls with compliant microspines, Proceedings of the International Conference on Advanced Robotics 2005 18-20

[196] S. Hirose, K. Yoneda, H. Tsukagoshi: TITAN VII: quadruped walking and manipulating robot on a steep slope, Proceedings of the IEEE International Conference on Robotics \& Automation 1997 494-500

[197] J. Bares, D. Wettergreen: Dante II: technical description, results and lessons learned, International Journal of Robotics Research 18(7), $621-$ 649 (1999)

[198] G. Endo, S. Hirose: Study on Roller-Walker (System Integration and Basic Experiments), Proceedings of the IEEE International Conference on Robotics \& Automation $19992032-2037$

[199] R. Siegwart, P. Lamon, T. Estier, M. Lauria, R. Piguet: Innovative design for wheeled locomotion in rough terrain, Robotics and Autonomous Systems 40, 151-162 (2002)

[200] K. Iagnemma, A. Rzepniewski, S. Dubowsky: Control of Robotic Vehicles with Actively Articulated Suspensions in Rough Terrain, Autonomous Robots 14,5-16 (2003) 
[201] K. Iagnemma, S. Dubowsky: Traction Control of Wheeled Robotic Vehicles in Rough Terrain with Application to Planetary Rovers, International Journal of Robotics Research 23(10-11), 1029-1040 (2004)

[202] C. Grand, F. Ben Amar, F. Plumet, P. Bidaud: Stability and traction optimization of a wheel-legged robot, International Journal of Robotics Research 23(10-11), $1041-1058$ (2004)

[203] G. Besseron, C. Grand, F. Ben Amar, F. Plumet, P. Bidaud: Locomotion modes of an hybrid wheel-legged robot, Proceedings of the International Conference on Climbing and Walking Robots 2004

[204] U. Saranli, M. Buehler, D.E. Koditschek: RHex - A Simple and Highly Mobile Hexapod Robot, International Journal of Robotics Research 20(7), $616-631$ (2001)

[205] T. Allen, R Quinn, R. Bachmann, R. Ritzmann: Abstracted Biological Principles Applied with Reduced Actuation Improve Mobility of Legged Vehicles, Proceedings of the IEEE/RSJ International Conference on Intelligent Robots \& Systems $20031370-1375$ 